\title{
The narrow road to data: Data sharing for global, unspecified reuse
}

\author{
Stefan Reichmann, TU Graz
}

This contribution has originally been conceived as the introduction to my dissertation, to be submitted in February 2022 to the University of Graz (Department of Sociology). In the dissertation, I take up the vast discourse on data sharing to argue that until now, the most influential positions (exemplified in the works of philosopher Sabina Leonelli and information scientists Christine L. Borgman) have construed their subject matter rather narrowly, both in terms of the dominant case studies as well as in focusing on the narrowly stated epistemic goals of the Open Science movement (of ensuring reproducibility, transparency, and data reuse, say). I take issue with both of these claims based on a series of empirical case studies, to show that data sharing is broader than has hitherto been assumed, especially in terms of its aims which have, by and large, been modelled along the lines of enabling unspecified, global data reuse. With this preprint, I explain what this means based on an analysis of three discourses, all pertaining to data sharing: Discussions predominantly found within information science documenting a tremendous variability of data types, formats, practices, and sharing behaviour, and an empirical literature that has frequently overlooked the difficulties in accounting for data practices at the level of faculties, research groups, departments, or universities, as well as a more critical discourse that has recently emerged around the factors that inhibit data sharing and reuse, such as the situatedness and contextuality of data practices. The contribution documents a growing awareness of the situated nature of data practices, in particular with respect to research activities in the global south. It further documents mounting evidence for what has been called data contextuality, discussions that ensued from the observation, already a decade old, that "data" are impossible to define, leading to a view now known as the relational view of data (data as potential evidence for knowledge claims). 


\section{Introduction: Data practices as an object of academic and policy interest}

\section{The recent discourse on data sharing and curation: Open Science and beyond}

(Empirical) Data are embedded in the core of our knowledge generation systems. They form the bedrock of what is deemed valuable information by (research) institutions (Richardson and Hoffman-Kim 2010), they are perceived as the arbiters of truth (Leonelli 2016) and as tools for enabling knowledge based on theory and observation (ibid. p. 69). Yet, despite their critical importance for the research enterprise, practices surrounding data production and management, such as "counting, calculating, cleaning, editing, extrapolating, ignoring, harmonizing, and so forth" (Cakici, Ruppert, and Scheel 2020) remain poorly understood in several dimensions. Based on conceptual and empirical work, this thesis seeks to describe core dimensions of (research) data practices, taking the Open Science movement and its core tenet of Open/FAIR Data (Wilkinson et al. 2016) as points of departure. Open Science forms an excellent starting point for this discussion: As an umbrella term, Open Science encompasses a multitude of assumptions about the future of knowledge production (Fecher and Friesike 2014). In addition to denoting a profound attempt at digital transformation of academia, Open Science promotes pervasive cultural, institutional, and infrastructural change (Mosconi et al. 2019). Although a decidedly sociotechnical process (Franzen 2018), Open Science has so far been conceived mainly as a technocratic project (Mirowski 2018), meaning that according to its critics, the more elusive social aspects of research practice have been overlooked by those committed to fostering Open Science practices. Open Science is very broad, envisioning transformation of all aspects of conducting research. In particular, advocates of Open Science have proposed reforms of institutions such as the peer review system) (Ross-Hellauer, Deppe, and Schmidt 2017) and the publication system (Suber 2012; Tennant, Jacques, and Collister 2016), scientific practices such as data sharing (Anagnostou et al. 2015; Andreoli-Versbach and Mueller-Langer 2014; Borgman 2015b; Duke and Porter 2013), and quality criteria, e.g., in terms of mandating reproducibility as a criterion for research quality ${ }^{1}$ (Academy of Medical Sciences 2015; Begley and loannidis 2015; Gilmore et al. 2017; Leonelli 2018). In the same vein, it has been suggested that the broader transformations of academia (digitization), likewise, pertain to all these aspects, with profound consequences for the conduct of scientific activity (Hey 2012).

Since the focus of this dissertation is on data sharing, I leave aside other aspects of Open Science, speaking to them only where they touch upon the topic of data sharing directly. There, significant efforts have gone into documenting the issues associated with making research data openly available, usually justified by appeal to greater reusability by researchers and by the general public, thereby making the research process more transparent and more efficient. At the same time, research institutions, libraries, funders and policy makers have devoted increasing amounts of time and resources to these issues as well, in terms of developing policies, infrastructures, and platforms to foster data sharing (Borgman 2018; Dulong de Rosnay 2017; Mauthner and Parry 2013). A growing number of national and international initiatives has consequently formed, with varying stakes and leverage in these efforts.

\footnotetext{
${ }^{1}$ A survey published in Science (Baker 2016) with 1500 respondents attempted to quantify what was then hailed as the crisis of reproducibility, by asking questions such as these: "How much published work in your field is reproducible?", "Have you failed to reproduce an experiment?", and "Have you ever tried to publish a reproducibility attempt?", to find that "[m]ore than $70 \%$ of researchers have tried and failed to reproduce another scientist's experiments, and more than half have failed to reproduce their own experiments" (p. 452). The publication marked a culmination point of calling out the so-called "crisis of reproducibility" by putting numbers to its extent. However, the survey does not offer an explication of the concept, nor does it seem to offer criteria for what actually counts as successful reproduction.
} 
Research funders have started to mandate research data management $\left(\mathrm{RDM}^{2}\right)$ to ensure the long-term value of research data, often through requiring grantees to submit Data Management Plans (DMPs) alongside research proposals (Mancilla et al. 2019; Mosconi et al. 2019). These efforts to streamline data practices have been condensed into the FAIR guiding principles for research data management (Wilkinson et al. 2016), a set of guidelines conceived by stakeholders from academia, industry, research funders and publishers (Mosconi et al. 2019:750). The FAIR guiding principles thereby constitute a very mature vision for RDM, mandating that research data be Findable, Accessible, Interoperable, and Reusable, thereby constituting a concise and measurable (Wilkinson et al. 2018) attempt to streamline disparate efforts to manage research data (Wilkinson et al. 2016). The FAIR guiding principles have been tremendously influential, having been adopted by the European Commission ${ }^{3}$ within its Horizon2020 and HorizonEurope funding schemes (Mayer et al. 2020), as well as other national and international research funders (in the case of Austria, the FWF for instance) and research-performing institutions in their efforts to institutionalize research data management. Given their fundamental importance for discussions of data sharing, it is worth to dwell upon the FAIR principles a little longer. As Wilkinson et al. (2016) report, the FAIR principles were developed in a workshop held in Leiden, Netherlands, in 2014 entitled 'Jointly Designing a Data Fairport', bringing together a wide group of academic and private stakeholders, "all of whom had an interest in overcoming data discovery and reuse obstacles". In fact, the stakeholders mostly had academic backgrounds in biomedical fields and the Life Sciences ${ }^{4}$. Workshop participants agreed upon "a minimal set of community-agreed guiding principles and practices" (Wilkinson et al. 2016). The aim of these principles was to enable all stakeholders to "more easily discover, access, appropriately integrate and re-use, and adequately cite, the vast quantities of information being generated by contemporary data-intensive science" (ibid.). The main outcome of the meeting was the by now familiar set of foundational principles subsequently elaborated in greater detail-namely, "that all research objects should be Findable, Accessible, Interoperable and Reusable (FAIR) both for machines and for people" (Wilkinson et al. 2016). The FAIR principles pertain to machine-readable data ${ }^{5}$ (Wilkinson et al. 2016), providing guidelines "for the publication of digital resources such as datasets, code, workflows, and research objects" ((Wilkinson et al. 2018). Indeed, the FAIR guiding principles have been so influential that their potentially idiosyncratic origins within a very specific research practice have been overlooked as they are being adopted as general guidelines for RDM by research institutions (Mancilla et al. 2019) and research funders. A large proportion of recent work on data practices (which previously had been a topic mainly for information scientists) has focused on precisely these fields, with Leonelli's landmark study on Data-centric Biology (Leonelli 2016) being a case in point.

\section{Data sharing and the digital transformation of academia}

Data sharing is but one example of the more general notion of "data practices" that comprises data curation, documentation, "packaging", and reuse. As Leonelli (2016) has convincingly argued, research data may be studied under many different dimensions, including epistemic (pertaining to knowledge production), economic (e.g. "data as the new oil"), political (in terms of, e.g. surveillance through Big Data, e.g. Zuboff 2019) as well as social aspects (e.g. in terms of labour that needs to be invested in data curation). A few of the most vexing problems surrounding the new agenda of data sharing (Fischer and Zigmond 2010) lie at the intersections of the economic, epistemic, political etc. aspects of research data. For instance,

\footnotetext{
2 "Research data management concerns the organization of data, from its entry to the research cycle through to the dissemination and archiving of valuable results. It aims to ensure reliable verification of results, and permits new and innovative research built on existing information." (Whyte and Tedds 2011) ${ }^{3} \mathrm{https}$ ://www.dtls.nl/2016/04/20/european-commission-allocates-e2-billion-to-make-research-data-fair/ ${ }^{4}$ https://www.datafairport.org/participants/index.html

${ }^{5}$ Findability refers to assigning a DOI (digital object identifier) to any digital research output (dataset, code, workflow, etc.); accessibility means retrievability of metadata using a standardized protocol, etc.(Wilkinson et al. 2016).
} 
it seems impossible to separate the economic value of data from their evidential (epistemic) value (Birch 2017). Through its emphasis on research data and other aspects of the research process, then, the Open Science movement throws into sharp relief what is currently happening to knowledge production (Franzen 2018), commodification of research outputs (Birch 2017, 2019), and academic labour relations (Manzi, Ojeda, and Hawkins 2019; Morley et al. 2018). Additionally, the digital transformation of academia itself does not happen in a vacuum. The broader transformations envisioned, and summarized, by the term Open Science take place within a decidedly neoliberal academia (Birch 2017; Münch 2014). These recent and distant transformations of academia need to be appreciated when trying to understand its digital transformation. As Rob Kitchin has noted:

"Much more critical attention needs to be paid to how open data projects are developing as complex sociotechnical systems...to date, efforts have concentrated on the political and technical work of establishing open data projects, and not enough on studying these discursive and material moves and their consequences" (Kitchin 2013).

Indeed, two of the most fundamental hopes associated with data sharing and data reuse concern the economic benefits of efficiency gains in research funding (Franzen 2018; Tennant et al. 2016) on the one hand, and institutionalised validation mechanisms for published findings to counteract the various crises of reproducibility (Begley and loannidis 2015; Leonelli 2018; Tennant et al. 2016), on the other. What has somewhat lagged behind these rapid developments is a confrontation of the various programmatic statements associated with Open Science (sometimes backed up by empirical ventures into what actually happens to research outputs) with the findings of theoretically informed sociology of science. There is a growing literature, to be sure, that attempts to come to (theoretical) terms with these developments. However, to paraphrase Kitchin (quoted above), much more critical attention is needed to understand how open (data) practices are implicated within various cultures and traditions of doing research. The present dissertation is an attempt to do just that. The following pages present its background and explain how the research papers comprising it are connected. They add to and critically engage with the recent plethora of RDM studies, engage with the limits of open data by way of a critical review of previous literature, and study the local practice of data handovers as a kind of data sharing strangely absent from accounts that build upon the idea of unspecified, global data reuse as the primary reason to share research data.

\section{What drives academic data sharing? (Unspecified) Data reuse is the primary goal}

The creation of research data has long been a topic of interest to social studies of science, e.g., in studies of the work that goes into creating "typical" representations of phenomena (Lynch 1985) and in the processes involved in deciding on the nature of concrete pieces of evidence (Amann and Knorr Cetina 1988). These studies concluded that unlike the term suggests, "data" result from complex processes of interpretation that are rarely described in published scientific work (Collins 1985; Knorr Cetina 1981; Lynch 1985). This feature of data practices has recently been taken up anew within the context of Open Science. Research into data journeys (Leonelli 2020), following extensive (ethnographic) work into the efforts and, indeed, work necessary, in terms of data curation and description, before data can be shared (Leonelli 2016). Indeed, recent years have seen increased interest on the part of researchers, research administrators and research funders in data practices (Anderson et al. 2007; Borghi and Van Gulick 2018; Chen and Wu 2017) and data governance (Burnette, Williams, and Imker 2016; Joshi and Krag 2010; Wilkinson et al. 2016). Data sharing is usually theorized, not as an end in itself (Pasquetto, Randles, and Borgman 2017), but as a prerequisite for data reuse (White et al. 2013). Yet, reuse is not yet well understood. At the very least, it requires data to be findable by potential users (Gregory et al. 2019). There is already a vast discussion on how and why research data are made available: For example, Tenopir et al. (2011) found that researchers were willing to reuse (and share) data provided they received credit for their datasets. Credit depends on the attributability of research results and datasets to 
individuals/institutions (Haak et al. 2020) and was found to be decisive as a reward mechanism for data creators as well as boosting the number of citations received (Piwowar, Day, and Fridsma 2007; Piwowar and Vision 2013a; Tenopir et al. 2011). Data reuse critically depends on the availability of data in public, well-curated databases (Wan and Pavlidis 2007). However, not all research data can or should be shared. Research outputs from third-party funded projects are rarely shareable (Banal-Estañol, Jofre-Bonet, and Lawson 2015), which puts limits on their reusability as well. In industry-funded projects, data sharing tends to be governed by agreements to account for industry partners' need for profit and researchers' need to publish (Myneni et al. 2016). Indeed, in industry-based research, the likelihood of sharing research data decreases with their competitive value (Haeussler 2011). In general, then, the literature finds that appropriate guidelines, policies, and standards are effective in fostering data sharing, data management, and reuse (Gregory 2020; Gregory, Cousijn, et al. 2020; Tenopir et al. 2011), all of them subject to disciplinary traditions, standards, and norms (Borgman 2012). The FAIR principles (Wilkinson et al. 2016) emphasise research data management and metadata, with appropriate tools for effective data management and curation being drivers for data sharing and reuse (Tenopir et al. 2011), as are appropriate disciplinary practices and routines (Borgman and Pasquetto 2017; Kim and Zhang 2015). Data sharing policies from research funders and publishers have been found particularly effective in fostering data sharing and reuse (Colavizza et al. 2020). Attitudes towards data sharing and reuse are mostly positive, though this is not necessarily reflected in data sharing and reuse practices (Tenopir et al. 2020).

An important overarching tenet of this thesis holds that these discourses surrounding data sharing - Open Science discourse, the vast literature studying research data management, as well as interest in data sharing broadly informed by social studies of science - have construed data sharing as releasing data for unspecified, global reuse. As a consequence, there is now a considerable body of literature studying data practices in a variety of settings and research fields that is largely concerned with the motivations of researchers (not) to share data (Vogeli et al. 2006). However, while this literature is empirically saturated, it largely takes data sharing and reuse for granted, thereby remaining, for the most part, theoretically unfounded and identifying barriers and facilitators to introducing and streamlining data sharing and reuse (e.g. in Borghi and Van Gulick 2018; Hsu et al. 2015; Tenopir et al. 2011). Indeed, while research data are produced in ever new ways (Hine 2016), a firm belief holds that the reuse of data created by others is beneficial for researchers as well as academia in general (Wilkinson et al. 2016). While the term "reuse" pertains to the entire spectrum of research outputs (observational, computational, experimental data, raw, derivative, or verified data, code, published results, protocols, and methodologies), here I shall be exclusively concerned with data reuse. An important reason for reusing research data is their recent exponential growth which entails an efficiency gain (Park and Park 2019). Sometimes, increase in data reuse is attributed to changing research practices (Murillo 2016) such as the increasing use of highthroughput technologies (Thanos 2017). Research institutions, funders and publishers are increasingly pushing the FAIR principles (Mancilla et al. 2019), promoting data reuse on epistemological, economical, and statistical grounds (Wade 2014). Although data sharing is not yet routine (Borgman 2012; Higman and Pinfield 2015; Wallis, Rolando, and Borgman 2013), it has been associated with numerous benefits, including economic growth (Custers and Bachlechner 2017), increased transparency (Leonelli, Spichtinger, and Prainsack 2015), enhanced integrity and reproducibility of research (Curty et al. 2017), as well as increased citations (Piwowar and Vision 2013b). Data release comes with a variety of rationales: Reproduction/verification of research, making publicly funded research publicly available, enabling others to ask new questions, and advancement of research (Borgman 2012:1066 f.; Pasquetto et al. 2017). Even though researchers' support for data reuse is almost unanimous (Gregory et al. 2020), to date, there are relatively few studies quantifying the extent of datareuse practices (Gregory 2020). In addition to assuming that data are generally shared, these studies assume, however tacitly, that data reuse will follow their availability (Borgman 2015a; Yoon et al. 2017); while data reuse in support of research is well-documented (Wallis et al. 2013), data reuse to drive new research is not (Gregory et al. 2020), mainly because data 
reuse is difficult to distinguish from data use (Borgman and Pasquetto 2017) and, because data reuse entails making the tacit dimensions of data generation explicit (Leonelli 2016). Reporting comprehensive results on data reuse practices and needs across a diverse set of disciplines and subfields, Gregory et al. (2019:422) helpfully distinguish between background (research support) and foreground uses of data (innovation).

\section{What drives data reuse?}

As we have seen, data sharing is predominantly justified by policy-makers, researchperforming institutions and researchers as a prerequisite for data reuse. The literature on data sharing and reuse represents a variety of disciplines and subdisciplines at different levels of granularity (Gregory, Groth, et al. 2020). Fields in the process of transitioning from fieldwork to automated data collection are of particular interest to scholars of data practices (Borgman 2015a; Edwards 2010; Wallis et al. 2013). Surveys studying data practices rely on qualitative methods and focus on cross-disciplinary or cross-national analyses of data sharing practices (Aydinoglu, Dogan, and Taskin 2017; Kim and Zhang 2015), with few specifically studying data reuse (Yoon 2017; Yoon et al. 2017; Yoon and Kim 2017). A variety of underlying factors have been identified, including funder policies (Piwowar and Chapman 2010; Piwowar et al. 2007), journal policies (Hanson, Sugden, and Alberts 2011) and archiving policies (Whitlock et al. 2010). Others have pointed towards psychological and behavioural factors (Curty 2015; Curty et al. 2017; Kim and Stanton 2016), ethical reasons (Fienberg, Martin and Straf 1985), findability (Gregory et al. 2019), data quality/relevance as perceived by potential users (Faniel et al. 2013); technological dimensions (Hine 2016; Zimmerman 2008), and disciplinary cultures (Borgman 2015a; Brown 2003; Faniel and Jacobsen 2010). Constraints on data sharing and reuse are often found to be endogenous to the research process. They include the complexity, specificities, and constraints of research designs as well as "human factor"-related reasons in relation to truth claims (Benestad et al. 2016). Culture, likewise, has been found to be a decisive factor in explaining the prevalence of data sharing and reuse (Borgman 2012). In some fields (the Life Sciences in particular, as documented extensively in, e.g., Leonelli 2016) data amounts grow fast, as does willingness to share and reuse them (Desjardins-Proulx et al. 2013; Leonelli et al. 2013; Park and Park 2019).

Barriers and drivers of data reuse have been described as technical (Gregory et al. 2020), economic and ethical (Bote and Termens 2019; Duke and Porter 2013; Poth 2019), legal (Dulong de Rosnay 2017), practical (Faniel and Jacobsen 2010), and cultural (Borgman 2015a). Studies document affinities between (data) reuse and willingness to share research outputs (Hsu et al. 2015; Joo, Kim, and Kim 2017; Kim and Zhang 2015; Yoon and Kim 2017, 2020). The needs of data users and the purposes of data reuse differ considerably across disciplines and data types (Gregory et al. 2019:422 f.). Among other factors, the (in)ability to trust data creators' methodological decisions constitutes a barrier to reuse (Faniel and Jacobsen 2010), especially for early career researchers (Faniel, Kriesberg, and Yakel 2012). In experimental settings with highly complex workflows, acquired data may be difficult to reuse (Foster et al. 2011:2184). Therefore, data reuse is challenging even within communities due to the highly sophisticated, context-dependent nature of research (Anderson et al. 2007; Borgman 2012, 2015a). Difficulties associated with determining reusability (Faniel and Jacobsen 2010; Faniel et al. 2012; Faniel, Kriesberg, and Yakel 2016) and relevance (Murillo 2019) of research outputs constitute a significant barrier to reuse and depend on the availability of metadata (i.e., information such as instrumentation and research methods used) (Murillo 2019). The ability to reuse data is predicated upon preservation of content as well as context (Leonelli 2016), where context information relevant for reuse includes information on data production, provenance, and terms of use (Faniel, Frank, and Yakel 2019). However, communicating the context of data generation to potential (re)users is challenging (Ambrósio et al. 2018; Faniel et al. 2019; Faniel and Yakel 2011; Yoon et al. 2017). This results in a need for mechanisms to measure the downstream utility of data (Fu et al. 2020). In the social sciences, data are reused when reuse is perceived to be useful (Goldstein 2018). Researchers from fields dealing with a diversity of data report challenges associated with data integration 
(Bowker 2000) and respective diversity in community (meta)data practices (Edwards et al. 2011). In many instances, data reuse is reduced to the technological dimension of designing data search systems (Gregory et al. 2019:459). 


\section{Unpacking data practices as aspects of the Open Science transition}

Indeed, the removal of access barriers is supposed to apply not only to publications (in the form of scientific articles, say). Rather, Open Science wishes to extend "transparency" to all kinds of research outputs, including research data. However,

"[m]uch of the vision behind [FAIR data respectively open data] presupposes that scientific data is inherently fungible, once a few pesky obstacles are cleared away. Some outstanding work by Leonelli (2016; Leonelli et al., 2015) has demonstrated that this impression concerning the nature of Open Data is illusory. Partisans of open science love to celebrate the kumbaya of 'data sharing'; Leonelli counters that there is no such thing. Data in the modern context would never venture outside the lab were it not for dedicated curators and their attached data consortia, such as the Open Biology Ontology Consortium (active since 2001). [...] The partisans of open science neglect to highlight the extent to which they define what the data actually signifies in Science 2.0, something that should give pause to anyone believing that data is effortlessly separable from its generators and curators." (Mirowski 2018:190 f.)

Aside from the loftier normative aspects of Open Science and how they hold up to scrutiny, the technical aspects of data sharing have been thoroughly examined. Excellent contributions by Leonelli and collaborators (Ankeny 2020; Bates, Lin, and Goodale 2016; Halfmann 2020; Karaca 2020; Leonelli 2016) suggest that the idea that data are inherently shareable if only we manage to remove certain obstacles is flawed; Leonelli's (2016) meticulous ethnographies show that data would rarely be shared beyond their context of origin were it not for the work of data curators.

This thesis takes issue with both the more affirmative strands of the Open Science discourse as well as with the vast empirical work studying data practices to suggest that they need to be considered as more than an object of technical and political governance with a view towards optimization (Fecher and Friesike 2014). It is thereby positioned well within and contributes to Open Science discourse. At the same time, however, it moves beyond this agenda in pointing out some of its shortcomings, specifically how certain aspects of the Open Science movement threaten the attainment of the movement's central aims. As Leonelli and colleagues note: "Finding ways to decide how sharing and transparency can be organised to be as fruitful as possible is one of the main challenges at present, while consensus that we need to share seems to have become solid" (Leonelli et al. 2015:2). The broader research aim thus concerns the kind of science envisioned by Open Science advocates and its compatibility with established research practices. With Fecher and Friesike (2014), an influential interpretation of Open Science holds that research processes can be optimized just like any other production process. Since a large proportion of the literature is predominantly affirmative, the question is timely for at least two reasons: Since Open Science is a movement but not a reality, there is scant empirical evidence as to its actual benefits. Open Science sometimes involves quite radical changes to current research practice. It is not clear whether such changes would in fact be beneficial (and for whom). Among other things, Open Science transports a conservative image of science along the lines of large, collaborative, industrialized research projects to the detriment of laboratory-based "little" science. To quote Mirowski (2018) once again:

"Indeed, it would even be misguided to infer that Science 2.0 [i.e. Open Science, S.R.] is being driven by some technological imperative to 'improve' science in any coherent sense; rather, it seeks to maximize data revelation as a means to its eventual monetization." (Mirowski 2018:193)

Whether or not Mirowski is right here, much closer attention needs to be paid to the role and function of data for the scientific enterprise while maintaining a critical distance to the attention data practices already receive from Open Science advocates. 


\title{
Methodological difficulties in studying data practices
}

\author{
Data sharing: What is the appropriate unit of analysis?
}

Data practices are notoriously hard to pin down as they frequently cut across research groups, departments, institutes, faculties, research fields, epistemic cultures, and other units of analysis (Wallis et al. 2013). However, as Mayernik (2016) points out, the unit of analysis determines whether or not we are able to find a "culture of data sharing". For the most part, the concept of data is left undefined (Kurata et al. 2017). "Data" denotes a heterogeneous class of objects; while the term originates in the Latin word for "given", the recognition that an observation, a measurement or a document constitutes data is itself an act of interpretation (Borgman 2012:1061), a conclusion that had already been drawn by earlier ventures into laboratory processes (Amann and Knorr Cetina 1988; Lynch 1985). This feature is exacerbated by the difficulty of empirically teasing apart data from practices of their production. Data types are often individuated, both by practitioners as well as scholars studying data practices, by reference to techniques of data collection/analysis. The research programme of studying data journeys (Leonelli 2020), with its associated relational view of data (Leonelli 2016), can be interpreted as an attempt to overcome these difficulties associated with defining data in the abstract by claiming that data can be anything that is taken as potential evidence for knowledge claims. Thylstrup (2019) relates this abstractness of data to their immateriality, employing the notion of "data traces" to emphasize that "data" denotes what is (un)intentionally left behind. In part, it is precisely their supposed immateriality that explains why data are simultaneously regarded as a processed good and expected to be malleable beyond the original production context (Gitelman 2013).

When it comes to assessing these claims, the sampling strategies employed by previous studies are instructive, as they offer a glimpse on how they have interpreted data (sharing) practices relative to modes of scientific organisation. These strategies range from (single) case studies relying heavily on ethnographic methods (interviews, participant observation) (Hsu et al. 2015; Leonelli 2016; Myneni et al. 2016; Wallis et al. 2013); qualitative small-N analyses (Kurata et al. 2017); quantitative studies of disciplinary data practices (Anderson et al. 2007; Borghi and Van Gulick 2018; Chen and Wu 2017); comparative studies within institutions (Akers and Doty 2013; Cox and Williamson 2015; Mancilla et al. 2019; Reichmann et al. 2021; Schöpfel et al. 2018); to large-scale national or international surveys (Aydinoglu et al. 2017; Chigwada, Chiparausha, and Kasiroori 2017; Elsayed and Saleh 2018; Koopman and Jager 2016; Liu and Ding 2016; Tenopir et al. 2011). The sampling strategies associated with these approaches correspond to (broadly) administrative versus (broadly) epistemic concerns, depending on the theoretical framework (research areas, specialties, disciplines, universities and departments) used. However, the organizational settings of research may not be best suited to the study of data practices, for several reasons: These units (e.g., disciplines) are frequently indistinguishable in terms of their social structure (Stichweh 1994). The related processes of discipline differentiation and integration combine cognitive inequality with structural equality (Stichweh 1994). The resulting units feature an internal communication context, a body of established knowledge (textbooks), current problems, methods/paradigms, career structures, and socialisation processes (ibid.). For studying data practices, they may be irrelevant as researchers "tend to work on problems, not in disciplines" (Klein 2000, 13). Indeed, many (applied) problems fall between disciplinary boundaries, stimulating reintegration which spawns new varieties of collaboration (multi-, inter-, and transdisciplinarity) (Maasen and Lieven 2006).

\section{Theoretical perspective: Epistemic cultures}

To avoid these conceptual aporias, the empirical research undertaken as part of this dissertation project has been heavily informed by an approach, already briefly mentioned above, developed by Karin Knorr Cetina (1999) to inquire into the social organization of science. I follow the understanding that the work of scientists is 
"organized into an overlapping and intersecting array of social and institutional arrangements. Scientists collaborate with one another and with citizens, students, technicians, practitioners, and other professionals in organizational settings that include disciplines, specialties, and research areas; for-profit, government, and nonprofit sectors; universities and departments; institutes and centers; invisible colleges and thought collectives; paradigms and epistemic cultures; research schools, groups, and teams; collaborations, laboratories, and collaboratories; social networks and social movements; boundary organizations, synthesis centers, and countless hybrids and variants of these" (Hackett et al. 2017:733).

In attempting to describe what separates data practices of different groups, I drew upon Knorr Cetina's work on epistemic cultures (Knorr Cetina 1999) as well as Collins' (1998) notion of evidential culture, as generative concepts, specifically when designing the interview instrument used for this thesis. "Epistemic cultures" were introduced by Karin Knorr Cetina (1999) in a comparative laboratory study, an approach to the sociology of science founded within the strong programme (Bloor 1991) that treats science as a social practice, as opposed to a purely cognitive, rational undertaking. While the sociology of science in the tradition of Merton (1973) had separated the social and cognitive norms of science to focus on the former (Zuckerman 1988), laboratory studies, drawing variously on relativist views from anthropology or the vastly influential writings of Kuhn (Kuhn 2012), studied how scientific knowledge is rooted in social life (Knorr Cetina 1991), seeking either to describe the processes by which scientific knowledge is produced in practice, variously by studying scientific controversies or to deconstruct the products (outputs) of science as products of culture (Knorr Cetina 1995; Doing 2008). These studies received a first systematic formulation in Latour and Woolgar's Laboratory Life (Latour and Woolgar 1986), which explored how researchers transform experiments into research outputs, which are then transformed into further resources to perform research (a mechanism they referred to as the cycle of credibility), thereby championing an analysis of researchers as members of an (alien) tribe. This perspective questioned rationalist accounts of the scientific process by suggesting that theory choice, experiments, etc., are not determined by logic (Bloor 1991) and evidence (Collins 1985). Instead, they (Knorr Cetina 1981; Latour 1994; Lynch 1985) produced detailed accounts of how scientists construct and justify knowledge claims. The notion of epistemic cultures is founded within comparative ethnographies of high-energy physics and microbiology, respectively (Knorr Cetina 1999), and refers to „interiorised processes of knowledge creation" (Knorr Cetina 2007: 363).

It was here employed as a sensitizing concept to suggest that data practices are implicated within these broader machineries of knowledge production (Knorr Cetina 2007). These lines of cultural separation are rooted in social separation (ibid. 364) founded in long phases of enculturation into disciplinary communities, which suggests that the variability of data practices can likewise be explained by reference to these enculturation processes. Important for our purposes, epistemic cultures are diverse in several dimensions: the meaning of "empirical" (Knorr Cetina 1999: $46 \mathrm{ff}$ and $79 \mathrm{ff}$.), the construction of the objects of knowledge (Knorr Cetina 1991), and what are taken to be the fundamental constituents of reality (Knorr Cetina 2007). The epistemic cultures framework allows for the analysis of all these in their relation to specific practices (Knorr Cetina 2007: 365), with the consequence that both the (supposed) variability as well as the contextuality of data (practices) are in fact artefacts of a specific (a)theoretical perspective.

\section{Definitions and analyses: Data as evidence for knowledge claims}

Indeed, the previous discussions of Open Science, data sharing, and reuse have been relatively devoid of (social) theory. In part, this is because the Open Science discourse is in fact (superficially) a-theoretical and descriptive, developing, at best, an implicit social theory of scientific practice ${ }^{6}$. Relatedly, taking many different forms and formats, data evade

\footnotetext{
${ }^{6}$ In this, the respective work bears a striking resemblance to the sociology of science in the Mertonian sense, where the cognitive (methodology) and social norms of science were kept separate (Merton 1973).
} 
theorization. Indeed, recent work on data practices has been criticized for avoiding questions of definition (Kurata et al. 2017). As has been pointed out by Leonelli, discussions of data management and sharing are traditionally unified by the assumption that data are (by and large) context-free representations of phenomena (Leonelli 2020:v) and that data can, at least in principle, be modelled and interpreted using statistical tools (Roth and Bowen 1999). Arguably, both assumptions still loom large, particularly in the recent hype around "big data" (Floridi 2012; Leonelli 2020), but have come under heavy criticism of late, mostly under the impression of the data sharing discourse, whence it has been found empirically that data, in fact, rarely leave their context of origin, a feature then attributed to their contextuality (Borgman 2012, 2015a). Where data do travel, this is usually courtesy of tremendous amounts of dedicated work (Leonelli 2016, 2017), with the consequence that research data sharing, in and of itself, would be illusory (Mirowski 2018) if it weren't for the efforts of data curators (e.g. Leonelli 2016, 2017). The labour needed to produce and make use of data to produce insights is an interesting issue in its own right. In this regard, important work has been produced on what Edwards and collaborators (Edwards et al. 2011) have termed "data friction", i.e. the conflictual role of (meta)data in (interdisciplinary) collaboration.

Data release has been theorized predominantly with a view towards their role as evidence for knowledge claims (Leonelli 2016:56). This discourse has construed researchers, in turn, as bound by scholarly altruism to share their data for the benefit of science (Lilja 2020). For instance, Borgman defines data as "entities used as evidence of phenomena for the purposes of research or scholarship" (Borgman 2015a:29) to stress how the characteristics as well as the meaning of data change with their context of production that Borgman typifies as follows: The meaning of data changes 1) with the specificity of the research purpose, 2) with the scope of data collection, and 3) with the goal of the research (Borgman 2012:1063). Such views assume, quite plausibly, that "data" is an umbrella term for any objects that can count as evidence for knowledge claims. Their common denominator, therefore, is their expected evidential value (Mayernik 2019). Data release has therefore been predominantly (though not exclusively) modelled along epistemic concerns (Borgman 2012:1066): Data are released either with a view towards reproducing or verifying published research, to enable others to ask new questions based on extant data, to make publicly funded research results publicly available, or to advance research in general. This way of framing data release incidentally explains the close attention, documented above, to data reuse in recent data scholarship (Gregory et al. 2020). However, while the view of data as potential evidence might be plausible with regards to resolving problems of definition, it cannot hope to denote all possible forms of data release since not all of them pertain to (unspecified) global data reuse. Likewise, the assumption seems to prolong the claim found within a significant portion of the literature (Mayernik, Batcheller, and Borgman 2011; Wallis et al. 2013) that data sharing is ultimately positive for the conduct of research.

\section{Getting hold of data: Variability and contextuality of data practices}

Data scholarship documents that intradisciplinary data practices are as varied as interdisciplinary practices (Borgman 2012; Wallis et al. 2013). The way data sharing objectives have typically been formulated tends to obscure these variations in research practices (Mauthner and Parry 2013) in the same way it obscures their context-based, situational, contingent, and pragmatic nature (Knorr Cetina 1999). While acknowledging the contextuality of data practices, most of the scholarship focusses on what separates disciplines, such as metadata standards, repositories, and data types. Where possible, the contextual information needed for data reuse is codified in metadata which contain "information necessary for investigators separated by space, time, institutions, or disciplinary norms to establish common ground" (Edwards et al. 2011:672). This has been taken to mean that "context" is ambiguous, as it refers both to "explicit" context (i.e., what is codified using metadata) or to "implicit" context (tacit knowledge, that which cannot be expressed/codified) (Kurata et al. 2017). What is deemed "context" and what is deemed "data" or "metadata" is itself the outcome of social processes that determine what is useful as evidence (Borgman 2015a; Leonelli 2016). Indeed, 
research activities have been found to depend on implicit knowledge that is not easily shared (often informally) between research sites and research communities (Collins 1985; Knorr Cetina 1999). The work that data require in order to be shared and reused has only been theorized much more recently (Leonelli et al. 2015; Mirowski 2018). Because the reusability of data depends on their interpretability (Kurata et al. 2017:3), data release requires making the tacit aspects of data handling explicit (Leonelli 2016). In this view, it is their context dependence that makes (global) data reuse difficult.

Structure versus culture - what happens at the level of OS policy, what happens at the level of research practices - data handovers as a middle ground;

Research strategy: interviews to capture dimensions of data practices

Survey to capture the structural aspects of the phenomenon - after extracting dimensions from interview data (data intensity, data curation, epistemic strategy) (typology of data practices to capture where propensity for sharing breaks down)

Theoretical perspective: epistemic cultures - avoid reification of data sharing as an a priori concern of researchers - issue is not with enabling reproducibility - connection between structure and agency, dimensions of data practices relate to aspects of epistemic cultures data sharing and OS as the enemy; respondents presented by faculty deans; Open Science as legitimating a specific kind of data practices, therefore resistance as it tends to amplify/reproduce inequalities between disciplines; resulting differences in the uptake (REFs) of Open practices

\section{Identification of some research gaps}

Taken together, the discussion above implies several research gaps: Discourse on data practices and sharing has frequently ignored the contextual nature of data practices (Hillyer et al. 2017); discourse on data practices has further been conducted in an atheoretical fashion, taking data sharing as an a priori condition for good scientific practice, or (and relatedly) it has sailed too close to the normative tenets of the Open Science movement in assuming that data sharing improves the conduct of research on multiple counts (Borgman 2012; Borgman and Pasquetto 2017), which has led a considerable proportion of the research to focus narrowly on those fields that subscribe to data sharing and reuse as normative ideals (Levin and Leonelli 2017; Perrier et al. 2017). As I have attempted to show, the discourse on data sharing has predominantly modelled the practice as a prerequisite for unspecified, global data reuse, spawning massive amounts of research into those aspects of data practices at odds with this general aim. Notably, these have been described in terms of the contextuality (Borgman 2012; Faniel et al. 2019; Kurata et al. 2017), the variability, and the relationality of data (practices). There is also a growing awareness, particularly among scholars studying data practices in the global south (L. Bezuidenhout et al. 2017; L. M. Bezuidenhout et al. 2017; Rappert and Bezuidenhout 2016), that data practices are highly situated, a feature that is difficult to account for based on quantitative data from the global north. Likewise, the close attention often paid to the broadly stated normative aims of Open Science has meant that the research programmes associated with studying data sharing have been formulated predominantly with a view towards enabling the practice in various contexts, thereby taking data sharing (and the narrowly stated epistemic goals of data reuse, reproducibility, and the like) as given. In addition, the empirical case studies have - by and large - treated precisely those fields where data sharing is already common practice (Wu and Worrall 2019). The focus on sharing for unspecified, global data reuse has likewise meant that important aspects of the practice have so far been overlooked. To be sure, much published work finds that data sharing is local in character (Borgman 2015a; Wallis et al. 2013), to go on asking why this should be so, and why not more research data are being shared beyond the local contexts of, e.g., research groups (see Borgman 2012 for an example), thereby (implicitly) taking unspecified, global reuse as a 
normative ideal of data sharing. This means that possibly, important aspects of the practice may have been missed.

As discussed above, this picture of data as context-free representations of underlying phenomena has been criticized on various counts. First, scholars have pointed out the difficulties associated with defining data in the abstract (Borgman 2012), thereby identifying a "conundrum" associated with sharing research data that consists in the apparent contradiction between widespread support for Open Science practices and the far smaller extent of actual data release (Borgman 2012). In part, this is due to methodical decisions of comparing data practices at the levels of research groups, disciplines, universities, and countries. In this context, it has already been proposed that data need a better definition in terms of their role as evidence. Second, the vast empirical engagement with what has come to be known as data journeys (following Leonelli's exemplary study in her 2016) has documented the tremendous amounts of work that are needed before data can travel, thereby throwing into question the idea that data can be understood as context-free representations of phenomena (Leonelli 2020). Indeed, Leonelli (2016) documents in minute detail the work (in terms of curation and explication of research procedures and practices) needed in advance of data sharing and reuse. Third, an increasing body of research documents the affordances associated with data release in the global south (Collyer 2018; Koopman and Jager 2016), documenting an increasing awareness of the situatedness (Leonelli 2017; Tkacz et al. 2021) and mutability of data practices. The discourse on research data management, and relatedly practices of data sharing and use, documents an astounding variability of data types, data amounts, data practices, and approaches to data sharing. The literature acknowledges variability, but - for the most part - does not attempt to find and explicate commonalities and differences in approaches, thereby remaining largely atheoretical. The contextuality of data (practices) (Kurata et al. 2017), discussed above, directly pertains to the tacit assumption throughout the literature on data sharing (and RDM) that the practice pertains to (is done in service of) the unspecified, global reuse of data. As is then documented extensively in various places, this condition is rarely met. In fact, data reuse is predicated upon making (certain) contextual aspects of data practices explicit, a precondition that has led to tremendous investments in some research fields (Leonelli 2017). As has been explained above, the relational view of data holds that data cannot be defined in the abstract, except as anything that can count as evidence for knowledge claims. In part, this attempt at conceptual clarification is a reaction towards the vast variability of data practices encountered across different research contexts.

Relatedly, the entire project of elucidating data practices (associated with different research programmes), it seems, has been formulated with a view towards the narrow epistemic goals of Open Science (to ensure transparency, reproducibility and data reuse, among others), and has thereby put too much emphasis on precisely those research fields where the practice, superficially at least, seems very mature (Mallasvik and Martins 2021). This comes with several implications: First, there may be forms of data release that cannot be modelled in terms of unspecified, global data reuse. Second, the a priori assumption that data release is ultimately beneficial for the conduct of research threatens to overlook other forms of research output that might be further removed from the production of datasets. Third, and relatedly, these research programmes come with implicit one-size-fits-all conceptions of data release (Levin and Leonelli 2017; Wu and Worrall 2019), an assumption at odds with the situated and contextual nature of data practices. In this, previous work has potentially modelled data sharing and reuse after the affordances associated with a specific way of conducting research. Taken together, this implies a narrow vision for data sharing, ultimately difficult to reconcile with the affordances of data practices in fields that are not a priori data-centric. 


\section{Conclusion}

\section{Data practices, data sharing, and Open Science}

One of the most salient aspects of recent work on data sharing and data practices more broadly concerns the staggering variability of practices and approaches it documents. As has been discussed throughout the engagement with the literature, this variability corresponds to a striking variety in the research fields, disciplines, and institutions under study. As a consequence, there is no common conceptual ground in the study of data practices (Kurata et al. 2017): For instance, the current state-of-the-art in RDM research has conceptualized data management predominantly as an a-historic, a-social practice, in most cases due to an unwarranted focus on technical problem solutions, both in terms of governance and software deployment (e.g. in Hunt and Bakker 2018; Littler et al. 2017). This perspective resonates with many earlier accounts of the role of data in the research enterprise from the philosophy of science where data have been conceived as impartial "arbiters of truth" (Leonelli 2016). In this, these influential treatments of data practices bear a striking resemblance to the first generation of sociologies of science in assuming, ever so tacitly, that the institutional aim of science is the pursuit of certified knowledge (Merton 1973) and that data sharing should be organized in service of that pursuit. This entailed a separation, epitomized within Robert Merton's The normative structure of science (Merton 1973), between the cognitive norms of science (methodologies, methods, etc.) and the social norms of science he famously identified as universalism, communalism, disinterestedness, and organized scepticism, with the associated belief that the institutional aim of science was to produce certified knowledge (ibid.). In the received discourse on data practices, this view corresponds to a striking preoccupation with the features of "good" data practices (Anagnostou et al. 2015; Chapman et al. 2014; Littler et al. 2017). The variability in data practices also corresponded, within the account presented in the preceding pages, to a rather eclectic strategy when presenting their most salient features and results, as well as a fundamental difficulty of presenting a common conceptual ground. The research agenda, however, bears striking resemblance to the early phases of the sociology of science and can - I argue - be criticized on similar grounds. Indeed, the most influential approaches to data sharing have either a) been carried out in an atheoretical fashion, mainly documenting variability and difficulties to define data in the abstract (Kurata et al. 2017), difficulties which gave rise to $b$ ) the research programme of studying data journeys (Leonelli 2020) with the corresponding relational theory of data (Leonelli 2016) that professes to study the curation, movement, and reuse of research data under a common conceptual framework. In important senses, both programmes have ventured too close to the normative tenets of the Open Science movement, assuming an a priori prevalence of sharing data for global unspecified reuse and taking data reuse largely for granted, at least as a normative ideal (Colavizza et al. 2020; Tenopir et al. 2011, 2020). This dissertation ventured on the same path for a time, documenting, as it does, variation in data practices based on studying a technical university, to find that respondents interpret data management as an administrative task, not necessarily part of good research practice. It also engaged critically with both discourses to suggest that the notion of data sharing is more complex than has hitherto been assumed, depending on the intended data reuse. As I attempted to show, based upon the interview material, data sharing is in important ways determined, not by generalized, global data reuse, but by specified, local reuse. As concerns the tenets of the Open Science movement, the supposed benefits of data sharing (again, predominantly interpreted as unspecified, global reuse) and data reuse are largely mediated by social factors. In what follows, the most salient findings are briefly summarized.

\section{Overview of findings}

\section{Growing appreciation for the situated and contextual nature of research data (practices)}

(Ross-Hellauer et al. 2022) show, based on an extensive and systematic review of Open Science discourse undertaken with a view towards potential threats to equity, that the initial 
optimism surrounding Open Science broadly and data sharing specifically (once a few obstacles have been removed) has more recently been replaced, somewhat, by growing appreciation of the situated nature of data sharing and reuse (REFs) and a growing awareness that one-size-fits-all policies risk privileging some disciplines. Indeed, there is increasing evidence to the effect that these (dis)advantages associated with data practices are cumulative as the exploitation of open data is strongly linked to available infrastructure and skills (RossHellauer et al. 2022).

\section{Data practices and Open Science: A priori conceptions of data sharing}

(Reichmann et al. 2021) document the large variability of data practices at a technical university. In retrospect, the discussions surrounding research data management are predicated upon a priori conceptions of data sharing as part of good scientific practice. The contribution took issue with this contention based upon the contention that our respondents took data management for a predominantly administrative activity, with little bearing on the quality of scholarly work. Likewise, the contribution documents that data practices exhibit both discipline-specific and discipline-invariant aspects. Similar to the discussions described in the introduction, this contribution found a staggering variability of data practices in terms of data types, amounts, and data sharing, with little or no apparent regularities. The other contributions, then, suggest that in order to describe regularities in data practices entails changing the unit of analysis, from research fields/institutions to the more abstract notion of epistemic culture (Knorr Cetina 2007).

\section{Unspecified, global reuse is not the primary aim of data release}

Further, data sharing has been theorized predominantly as unspecified, global data reuse, thereby largely failing to appreciate the importance of local forms of data release, theorized in terms of data handovers to ensure the continuity of research work. The necessity to ensure local data release is explicated by reference to increasing academic mobility to suggest that where influential accounts have spoken to the difficulties implicated in ensuring data mobility, the underlying problems associated with data sharing actually pertain to ensuring the continuity of research projects in view of increasing staff turnover. This was not to suggest that research managers and administrators are actively repurposing research data management to strategically control institutional dispositions over data through asking researchers to explicate (as analysed in Leonelli 2016) the tacit dimensions of data handling; the contribution merely suggests that where influential positions on data sharing posit ensuring reproducibility and reusability of data as the primary motivations, the contribution details how data release is often much more closely aligned with the problems of local data reuse.

\section{Data practices and epistemic cultures}

The perspective of epistemic cultures, associated most closely with the work of Karin Knorr Cetina (Knorr Cetina 1991, 1999, 2007), suggests that data practices need to be theorized with respect to more general assumptions about research aims and processes instead of taking broadly administrative categories (discipline, faculty, research group, university) at face value. As the examples discussed in the paper show, these assumptions typically vary between research fields, along multiple dimensions. For instance, the way research fields stabilize and secure their phenomena of interest (Bogen and Woodward 1988; Collins 1985, 1994) - a feature that looms large in the recent interest in reproducible research (Leonelli 2018) - is intimately tied to epistemic strategies (Knorr Cetina 1999). Whether data preservation, sharing, and reuse are meaningful relative to a given epistemic strategy, then, depends on the techniques used to stabilize phenomena. In some instances, this is done via constructing datasets. In some fields this is achieved differently, for instance, through 
constructing prototypes. This suggests that theories of data sharing and reuse need to broaden their outlook to include research contexts where other strategies of stabilization abound.

\section{Outlook}

In summary, the account developed within the preceding pages corresponds in important respects to the first wave of laboratory studies (Bloor 1976) in that it criticizes the data sharing discourse on the same counts: for tacitly assuming an a-theoretical, a-historic, a priori conception of data sharing (even while superficially tending to the practices associated with it), and relatedly, for largely failing to move beyond the narrowly stated epistemic aims of the Open Science movement of ensuring transparency, reproducibility and data reuse, thereby overemphasising those research fields where the practice, superficially at least, seems very mature. This predicament comes with several implications: First, there may be other forms of data release that cannot be modelled in terms of unspecified, global data reuse. Second, the a priori assumption that data release is ultimately beneficial for the conduct of research threatens to overlook other forms of research output that might be further removed from the production of datasets. Third, and relatedly, these research programmes come with implicit one-size-fits-all conceptions of data release (Levin and Leonelli 2017; Wu and Worrall 2019), an assumption at odds with the situated and contextual nature of data practices. In this, previous work has potentially modelled data sharing and reuse after the affordances associated with a specific way of conducting research. Taken together, this implies a narrow vision for data sharing, ultimately difficult to reconcile with the affordances of data practices in fields that are not a priori data-centric (Leonelli 2016).

Studying data sharing as an issue spanning multiple discourses and approaches, the dissertation found that 1) the concept denotes but a special case of the broader notion of data release, a concept that comprises data sharing as unspecified, global release, as well as data handovers, as the specified, local release of data, 2) that the three influential discourses studying data practices (Open Science, information science, STS) all venture close to a normative reading of data sharing and reuse, as something to be encouraged, and 3), that data sharing needs yet to be unpacked as part of the broader research process. In fact, the most influential strands of data sharing discourse seem to be built upon empirical material from data-centric disciplines (a feature that is already, if somewhat quietly, acknowledged in calls for speaking about Open Research instead of Open Science); the empirical materials presented within these pages point to the fact that not only are there epistemic cultures that are inherently more suited to data-centric research practices; it seems that the most influential accounts of data practices have been modelled after data-centric fields, which threatens to leave out a large class of data practices. In fact, Leonelli's (2016) monumental study is called "data-centric biology", which might already hold the clue that other research fields might simply not be data-centric in the relevant sense. As I tried to show, the notion of data-centric research should be unpacked by reference to the idea of curation. Considering data practices as parts of epistemic cultures (Knorr Cetina 2007) opens up the possibility that data practices are associated with vastly divergent epistemic goals. An important learning from this research concerns the apparently chaotic nature of data practices, as documented in the vast literature studying RDM. However, this impression is somewhat deceptive (even if influential) (Mayernik 2016). When it comes to studying data practices, picking the unit of analysis is decisive. 


\section{Limitations}

This project has been conceived as an attempt to synthesise, based on recent (and distant) science studies discourse on data practices, a disparate set of administrative and research projects into aspects of the Open Science transition with a view towards elucidating some aspects of the recent (and equally disparate) discourse surrounding data sharing. This has meant that the inspiration, concepts, and data upon which these contributions are based come from different places and were collected with different aims in mind. In other words, other than a broad engagement with Open Science and the fact that the associated work was done largely within the same research group, there is no conceptual unity to these endeavours. I have attempted to show, however, based upon a detailed analysis of three interrelated discourses associated broadly with the topic of data sharing, that a common denominator can be found in certain assumptions with respect to the concept of data, critical to all three discourses, that I have been engaging with and criticising in the preceding pages. Relatedly, it should be noted that unlike influential work on data practices, this study did not attempt an ethnographic design (which would have been warranted given the contextuality and, indeed, intractability of research data), but resorted to literature reviews, surveys, and grounded-theory driven analyses of interview materials to enter into a productive dialogue between empirical materials, the three discourses described in detail above, and equally ethnography-informed perspectives on scientific practices. The aim of this approach was to point out, through adding incrementally to each of the discourses, where our understanding of data practices is still indebted to certain (questionable) assumptions. 


\section{References}

Academy of Medical Sciences. 2015. Reproducibility and Reliability of Biomedical Research: Improving Research Practice.

Akers, Katherine G., and Jennifer Doty. 2013. 'Disciplinary Differences in Faculty Research Data Management Practices and Perspectives'. International Journal of Digital Curation 8(2):5-26. doi: 10.2218/ijdc.v8i2.263.

Albornoz, Denisse, Maggie Huang, Issra Marie Martin, Maria Mateus, Aicha Yasmine Touré, and Leslie Chan. 2018. 'Framing Power: Tracing Key Discourses in Open Science Policies'. Toronto: 22nd International Conference on Electronic Publishing - Connecting the Knowledge Commons: From Projects to Sustainable Infrastructure, ELPUB 2018.

Albornoz, Denisse, Alejandro Posada, Angela Okune, Rebecca Hillyer, and Leslie Chan. 2017. Co-Constructing an Open and Collaborative Manifesto to Reclaim the Open Science Narrative. edited by L. Chan and F. Loizides.

Amann, Klaus, and Karin Knorr Cetina. 1988. 'The Fixation of (Visual) Evidence'. Human Studies 11(2):133-69. doi: 10.1007/BF00177302.

Ambrósio, Lenita M., José Maria N. David, Regina Braga, Fernanda Campos, Victor Ströele, and Marco Antônio Araújo. 2018. 'Using Context Elements and Data Provenance to Support Reuse in Scientific Software Ecosystem Platform': Pp. 255-62 in Proceedings of the 20th International Conference on Enterprise Information Systems. Funchal, Madeira, Portugal: SCITEPRESS - Science and Technology Publications.

Amorim, Ricardo Carvalho, Joao Aguiar Castro, Joao Rocha da Silva, and Cristina Ribeiro. 2017. 'A Comparison of Research Data Management Platforms: Architecture, Flexible Metadata and Interoperability'. Universal Access in the Information Society 16(4):85162. doi: 10.1007/s10209-016-0475-y.

Anagnostou, Paolo, Marco Capocasa, Nicola Milia, Emanuele Sanna, Cinzia Battaggia, Daniela Luzi, and Giovanni Destro Bisol. 2015. 'When Data Sharing Gets Close to 100\%: What Human Paleogenetics Can Teach the Open Science Movement.' PLoS ONE 10(3):e0121409.

Anderson, Nicholas R., E. Sally Lee, J. Scott Brockenbrough, and Mark E. Minie. 2007. 'Issues in Biomedical Research Data Management and Analysis: Needs and Barriers'. Journal of the American Medical Informatics Association: JAMIA 14(4):478-88. doi: 10.1197/jamia.M2114.

Andreoli-Versbach, Patrick, and Frank Mueller-Langer. 2014. 'Open Access to Data: An Ideal Professed but Not Practised'. Research Policy 43(9):1621-33. doi: 10.1016/j.respol.2014.04.008.

Ankeny, Rachel A. 2020. 'Tracing Data Journeys Through Medical Case Reports: Conceptualizing Case Reports Not as "Anecdotes" but Productive Epistemic Constructs, or Why Zebras Can Be Useful'. Pp. 59-76 in Data Journeys in the Sciences, edited by S. Leonelli and N. Tempini. Cham: Springer International Publishing.

Aula, Ville. 2019. 'Institutions, Infrastructures, and Data Friction - Reforming Secondary Use of Health Data in Finland'. Big Data \& Society 6(2):2053951719875980. doi: $10.1177 / 2053951719875980$. 
Aydinoglu, Arsev Umur, Guleda Dogan, and Zehra Taskin. 2017. 'Research Data Management in Turkey: Perceptions and Practices'. Library Hi Tech 35(2):271-89. doi: 10.1108/LHT11-2016-0134.

Baker, Monya. 2016. '1,500 Scientists Lift the Lid on Reproducibility'. Nature News 533(7604):452. doi: 10.1038/533452a.

Banal-Estañol, Albert, Mireia Jofre-Bonet, and Cornelia Lawson. 2015. 'The Double-Edged Sword of Industry Collaboration: Evidence from Engineering Academics in the UK'. Research Policy 44(6):1160-75. doi: 10.1016/j.respol.2015.02.006.

Bardi, Alessia, Vittore Casarosa, and Paolo Manghi. 2018. 'The European Project OpenUP: OPENing UP New Methods, Indicators and Tools for Peer Review, Impact Measurement and Dissemination of Research Results'. Pp. 240-48 in Digital Libraries and Multimedia Archives, Ircdl 2018. Vol. 806, edited by G. Serra and C. Tasso. Berlin: Springer-Verlag Berlin.

Bates, Jo. 2018. 'The Politics of Data Friction'. Journal of Documentation. doi: 10.1108/JD-052017-0080.

Bates, Jo, and Paula Goodale. 2017. 'Making Data Flow for the Climate Risk Market'. Television \& New Media 18(8):753-68. doi: 10.1177/1527476416677114.

Bates, Jo, Yu-Wei Lin, and Paula Goodale. 2016. 'Data Journeys: Capturing the Socio-Material Constitution of Data Objects and Flows'. Big Data \& Society 3(2):2053951716654502. doi: $10.1177 / 2053951716654502$.

Beck, Jeffrey, Kathryn Funk, Melissa Harrison, Jo McEntyre, Josie Breen, Andy Collings, Paul Donohoe, Michael Evans, Louisa Flintoft, Audrey Hamelers, Phil Hurst, Thomas Lemberger, Jennifer Lin, Niamh O'Connor, Michael Parkin, Sam Parker, Peter Rodgers, Magdalena Skipper, and Michael Stoner. 2018. 'Publishing Peer Review Materials [Version 1; Referees: 2 Approved]'. F1000Research 7.

Begley, C. Glenn, and John P. A. Ioannidis. 2015. 'Reproducibility in Science: Improving the Standard for Basic and Preclinical Research'. Circulation Research 116(1):116-26. doi: 10.1161/CIRCRESAHA.114.303819.

Benestad, Rasmus E., Dana Nuccitelli, Stephan Lewandowsky, Katharine Hayhoe, Hans Olav Hygen, Rob van Dorland, and John Cook. 2016. 'Learning from Mistakes in Climate Research'. Theoretical and Applied Climatology 126(3):699-703. doi: 10.1007/s00704015-1597-5.

Bezuidenhout, Louise, Ann H. Kelly, Sabina Leonelli, and Brian Rappert. 2017. ' \$100 Is Not Much To You': Open Science and Neglected Accessibilities for Scientific Research in Africa'. Critical Public Health 27(1):39-49. doi: 10.1080/09581596.2016.1252832.

Bezuidenhout, Louise M., Sabina Leonelli, Ann H. Kelly, and Brian Rappert. 2017. 'Beyond the Digital Divide: Towards a Situated Approach to Open Data'. Science and Public Policy 44(4):464-75. doi: 10.1093/scipol/scw036.

Birch, Kean. 2017. 'Rethinking Value in the Bio-Economy: Finance, Assetization, and the Management of Value'. Science, Technology, \& Human Values 42(3):460-90. doi: $10.1177 / 0162243916661633$.

Birch, Kean. 2019. 'Technoscience Rent: Toward a Theory of Rentiership for Technoscientific Capitalism': Science, Technology, \& Human Values. doi: 10.1177/0162243919829567. 
Birch, Kean, and David Tyfield. 2013. 'Theorizing the Bioeconomy: Biovalue, Biocapital, Bioeconomics or . . What?' Science, Technology, \& Human Values 38(3):299-327. doi: $10.1177 / 0162243912442398$.

Bloor, David. 1976. Knowledge and Social Imagery. 2nd ed. Chicago: Chicago University Press.

Bogen, James, and James Woodward. 1988. 'Saving the Phenomena'. The Philosophical Review 97(3):303-52.

Bonde Thylstrup, Nanna, Mikkel Flyverbom, and Rasmus Helles. 2019. 'Datafied Knowledge Production: Introduction to the Special Theme'. Big Data \& Society 6(2):2053951719875985. doi: 10.1177/2053951719875985.

Borghi, John A., and Ana E. Van Gulick. 2018. 'Data Management and Sharing in Neuroimaging: Practices and Perceptions of MRI Researchers'. PloS One 13(7):e0200562.

Borgman, Christine L. 2012. 'The Conundrum of Sharing Research Data'. Journal of the American Society for Information Science and Technology 63(6):1059-78. doi: 10.1002/asi.22634.

Borgman, Christine L. 2015a. Big Data, Little Data, No Data: Scholarship in the Networked World. Cambridge, MA, London: The MIT Press.

Borgman, Christine L. 2015b. 'If Data Sharing Is the Answer, What Is the Question?' Ercim News (100):15-16.

Borgman, Christine L. 2018. 'Data Sharing and Reuse in Interdisciplinary Scientific Collaborations: Challenges of Heterogeneous Practice'.

Borgman, Christine L., and Irene V. Pasquetto. 2017. 'Why Data Sharing and Reuse Are Hard To Do'.

Bote, Juan-Jose, and Miquel Termens. 2019. 'Reusing Data: Technical and Ethical Challenges'. Desidoc Journal of Library \& Information Technology 39(6):329-37. doi: 10.14429/djlit.39.6.14807.

Boumans, Marcel, and Sabina Leonelli. 2020. 'From Dirty Data to Tidy Facts: Clustering Practices in Plant Phenomics and Business Cycle Analysis'. Pp. 79-101 in Data Journeys in the Sciences, edited by S. Leonelli and N. Tempini. Cham: Springer International Publishing.

Bourdieu, Pierre. 1975. 'The Specificity of the Scientific Field and the Social Conditions of the Progress of Reason'. Social Science Information 14(6):19-47. doi: 10.1177/053901847501400602.

Brown, Cecelia. 2003. 'The Role of Electronic Preprints in Chemical Communication: Analysis of Citation, Usage, and Acceptance in the Journal Literature'. Journal of the American Society for Information Science and Technology 54(5):362-71. doi: 10.1002/asi.10223.

Bugaje, Maryam, and Gobinda Chowdhury. 2018. 'Identifying Design Requirements of a UserCentered Research Data Management System'. Pp. 335-47 in Lecture Notes in Computer Science, Information Systems and Applications, incl. Internet/Web, and $\mathrm{HCl}$. Vol. 11279. 
Burnette, Margaret, Sarah Williams, and Heidi Imker. 2016. 'From Plan to Action: Successful Data Management Plan Implementation in a Multidisciplinary Project'. Journal of EScience Librarianship 5(1):e1101. doi: 10.7191/jeslib.2016.1101.

Cakici, Baki, Evelyn Ruppert, and Stephan Scheel. 2020. 'Peopling Europe through Data Practices: Introduction to the Special Issue'. Science, Technology, \& Human Values 45(2):199-211. doi: 10.1177/0162243919897822.

Cambrosio, Alberto, Jonah Campbell, Etienne Vignola-Gagné, Peter Keating, Bertrand R. Jordan, and Pascale Bourret. 2020. "'Overcoming the Bottleneck": Knowledge Architectures for Genomic Data Interpretation in Oncology'. Pp. 305-27 in Data Journeys in the Sciences, edited by S. Leonelli and N. Tempini. Cham: Springer International Publishing.

Canaan, Joyce E., and Wesley Shumar. 2008. Structure and Agency in the Neoliberal University. Routledge.

Carroll, Michael W. 2015. 'Sharing Research Data and Intellectual Property Law: A Primer'. PLOS Biology 13(8):e1002235. doi: 10.1371/journal.pbio.1002235.

Carrozza, Tomás Javier, and Susana Silvia Brieva. 2015. 'Who Owns the Atoms? Nanotechnology and Intellectual Property Rights'. Íconos 19(52):163-80.

Caso, Roberto, and Rossana Ducato. 2014. Intellectual Property, Open Science and Research Biobanks. SSRN Scholarly Paper. ID 2511602. Rochester, NY: Social Science Research Network.

Chapman, S. J., B. Shelton, H. Mahmood, J. E. F. Fitzgerald, E. Harrison, and A. Bhangu. 2014. 'Promoting Transparency in Clinical Research: Systematic Review of Disclosure and Data-Sharing Policies in Surgical Journals'. International Journal of Surgery 12:S52. doi: 10.1016/j.jisu.2014.07.238.

Chen, Xiujuan, and Ming Wu. 2017. 'Survey on the Needs for Chemistry Research Data Management and Sharing'. The Journal of Academic Librarianship 43(4):346-53. doi: 10.1016/j.acalib.2017.06.006.

Chigwada, Josiline, Blessing Chiparausha, and Justice Kasiroori. 2017. 'Research Data Management in Research Institutions in Zimbabwe'. Data Science Journal 16:1-9. doi: $10.5334 / d s j-2017-031$.

Cinnamon, J. 2020. 'Data Inequalities and Why They Matter for Development'. Information Technology for Development 26(2):214-33. doi: 10.1080/02681102.2019.1650244.

Clark, Burton R. 1998. 'The Entrepreneurial University: Demand and Response'. Tertiary Education and Management 4(1):5-16. doi: 10.1007/BF02679392.

Colavizza, Giovanni, lain Hrynaszkiewicz, Isla Staden, Kirstie Whitaker, and Barbara McGillivray. 2020. 'The Citation Advantage of Linking Publications to Research Data' edited by J. M. Wicherts. PLOS ONE 15(4):e0230416. doi: 10.1371/journal.pone.0230416.

Collins, Harry M. 1985. Changing Order. Replication and Induction in Scientific Practice. 1st ed. London, Beverly Hills, New Delhi: SAGE Publications.

Collins, Randall. 1994. 'Why the Social Sciences Won't Become High-Consensus, RapidDiscovery Science'. Sociological Forum 9(2):155-77. doi: 10.1007/BF01476360. 
Collyer, Fran M. 2018. 'Global Patterns in the Publishing of Academic Knowledge: Global North, Global South'. Current Sociology 66(1):56-73. doi: 10.1177/0011392116680020.

Coopmans, Catelijne, and Brian Rappert. 2020. 'Data Journeys in Art? Warranting and Witnessing the "Fake" and the "Real" in Art Authentication'. Pp. 371-88 in Data Journeys in the Sciences, edited by S. Leonelli and N. Tempini. Cham: Springer International Publishing.

Cox, Andrew M., Mary Anne Kennan, Liz Lyon, and Stephen Pinfield. 2017. 'Developments in Research Data Management in Academic Libraries: Towards an Understanding of Research Data Service Maturity'. Journal of the Association for Information Science and Technology 68(9):2182-2200. doi: 10.1002/asi.23781.

Cox, Andrew, and Laurian Williamson. 2015. 'The 2014 DAF Survey at the University of Sheffield'. International Journal of Digital Curation 10(1):210-29. doi: 10.2218/ijdc.v10i1.362.

Curty, Renata. 2015. "Beyond "Data Thrifting": An Investigation of Factors Influencing Research Data Reuse In the Social Sciences'. Dissertations - ALL.

Curty, Renata Gonçalves, Kevin Crowston, Alison Specht, Bruce W. Grant, and Elizabeth D. Dalton. 2017. 'Attitudes and Norms Affecting Scientists' Data Reuse' edited by C. R. Sugimoto. PLOS ONE 12(12):e0189288. doi: 10.1371/journal.pone.0189288.

Custers, Bart, and Daniel Bachlechner. 2017. 'Advancing the EU Data Economy: Conditions for Realizing the Full Potential of Data Reuse'. Information Polity 22(4):291-309. doi: 10.3233/IP-170419.

Desjardins-Proulx, Philippe, Ethan P. White, Joel J. Adamson, and Karthik Ram. 2013. 'The Case for Open Preprints in Biology'. PLoS Biology 11(5). doi: 10.1371/journal.pbio.1001563.

DiPrete, Thomas A., and Gregory M. Eirich. 2006. 'Cumulative Advantage as a Mechanism for Inequality: A Review of Theoretical and Empirical Developments'. Annual Review of Sociology 32(1):271-97. doi: 10.1146/annurev.soc.32.061604.123127.

Doing, Park. 2008. 'Give Me a Laboratory and I Will Raise a Discipline: The Past, Present, and Future Politics of Laboratory Studies in STS'. Pp. 279-95 in.

Duke, Clifford S., and John H. Porter. 2013. 'The Ethics of Data Sharing and Reuse in Biology'. BioScience 63(6):483-89. doi: 10.1525/bio.2013.63.6.10.

Dulong de Rosnay, Mélanie. 2017. 'The legal and policy framework for scientific data sharing, mining and reuse'. in Ouvrir, partager, réutiliser, edited by C. Mabi, J.-C. Plantin, and L. Monnoyer-Smith. Éditions de la Maison des sciences de l'homme.

Edwards, Paul N. 2010. A Vast Machine. Computer Models, Climate Data, and the Politics of Global Warming. London, Cambrige, MA: The MIT Press.

Edwards, Paul N., Matthew S. Mayernik, Archer L. Batcheller, Geoffrey C. Bowker, and Christine L. Borgman. 2011. 'Science Friction: Data, Metadata, and Collaboration'. Social Studies of Science 41(5):667-90. doi: 10.1177/0306312711413314. 
Elsayed, Amany M., and Emad I. Saleh. 2018. 'Research Data Management and Sharing among Researchers in Arab Universities: An Exploratory Study'. IFLA Journal 44(4):281-99. doi: 10.1177/0340035218785196.

Etzkowitz, Henry. 1990. 'The Capitalization of Knowledge: The Decentralization of United States Industrial and Science Policy from Washington to the States'. Theory and Society 19(1):107-21.

Faniel, Ixchel, Eric Kansa, Sarah Whitcher Kansa, Julianna Barrera-Gomez, and Elizabeth Yakel. 2013. 'The Challenges of Digging Data: A Study of Context in Archaeological Data Reuse'. Pp. 295-304 in Jcdl'13: Proceedings of the 13th Acm/leee-Cs Joint Conference on Digital Libraries. New York: Assoc Computing Machinery.

Faniel, Ixchel M., Rebecca D. Frank, and Elizabeth Yakel. 2019. 'Context from the Data Reuser's Point of View'. Journal of Documentation 75(6):1274-97. doi: 10.1108/JD-082018-0133.

Faniel, Ixchel M., and Trond E. Jacobsen. 2010. 'Reusing Scientific Data: How Earthquake Engineering Researchers Assess the Reusability of Colleagues' Data'. Computer Supported Cooperative Work-the Journal of Collaborative Computing and Work Practices 19(3-4):355-75. doi: 10.1007/s10606-010-9117-8.

Faniel, Ixchel M., Adam Kriesberg, and Elizabeth Yakel. 2012. 'Data Reuse and Sensemaking among Novice Social Scientists: Data Reuse and Sensemaking among Novice Social Scientists'. Proceedings of the American Society for Information Science and Technology 49(1):1-10. doi: 10.1002/meet.14504901068.

Faniel, Ixchel M., Adam Kriesberg, and Elizabeth Yakel. 2016. 'Social Scientists' Satisfaction with Data Reuse'. Journal of the Association for Information Science and Technology 67(6):1404-16. doi: 10.1002/asi.23480.

Faniel, Ixchel M., and Elizabeth Yakel. 2011. 'Significant Properties as Contextual Metadata'. Journal of Library Metadata 11(3-4):155-65. doi: 10.1080/19386389.2011.629959.

Fecher, Benedikt, and Sascha Friesike. 2014. 'Open Science: One Term, Five Schools of Thought'. Pp. 17-48 in Opening Science. The Evolving Guide on How the Internet is Changing Research, Collaboration and Scholarly Publishing. Cham, Heidelberg, New York, Dordrecht, London: Springer.

Fell, Michael J. 2019. 'The Economic Impacts of Open Science: A Rapid Evidence Assessment'. Publications 7(3):46. doi: 10.3390/publications7030046.

Fischer, Beth A., and Michael J. Zigmond. 2010. 'The Essential Nature of Sharing in Science'. Science and Engineering Ethics 16(4):783-99. doi: 10.1007/s11948-010-9239-x.

Fleck, Christian. 2013. 'Impact Factor Fetishism'. European Journal of Sociology 2:327-56. doi: $10.1017 /$ S0003975613000167.

Floridi, Luciano. 2012. 'Big Data and Their Epistemological Challenge'. Philosophy \& Technology 25(4):435-37. doi: 10.1007/s13347-012-0093-4.

Foster, Joseph M., Sven Degroeve, Laurent Gatto, Matthieu Visser, Rui Wang, Johannes Griss, Rolf Apweiler, and Lennart Martens. 2011. 'A Posteriori Quality Control for the Curation and Reuse of Public Proteomics Data'. Proteomics 11(11):2182-94. doi: 10.1002/pmic.201000602. 
Franzen, Martina. 2018. 'Die Digitale Transformation Der Wissenschaft'. Beiträge Zur Hochschulforschung 40(4):8-28.

Fu, Jingxin, Karen Li, Wubing Zhang, Changxin Wan, Jing Zhang, Peng Jiang, and X. Shirley Liu. 2020. 'Large-Scale Public Data Reuse to Model Immunotherapy Response and Resistance'. Genome Medicine 12(1):21. doi: 10.1186/s13073-020-0721-z.

Giffels, Joe. 2010. 'Sharing Data Is a Shared Responsibility'. Science and Engineering Ethics 16(4):801-3. doi: 10.1007/s11948-010-9230-6.

Gilmore, Rick O., Michele T. Diaz, Brad A. Wyble, and Tal Yarkoni. 2017. 'Progress toward Openness, Transparency, and Reproducibility in Cognitive Neuroscience'. Annals of the New York Academy of Sciences 1396(1):5-18. doi: 10.1111/nyas.13325.

Gitelman, Lisa. 2013. 'Raw Data' Is an Oxymoron. Cambridge MA; London: The MIT Press.

Goldstein, Scott. 2018. 'Social Scientists' Data Reuse Principally Influenced by Disciplinary Norms, Attitude, and Perceived Effort'. Evidence Based Library and Information Practice 13(2):109-11. doi: 10.18438/eblip29415.

Grahe, Jon E., Kelly Cuccolo, Dana C. Leighton, and Leslie D. Cramblet Alvarez. 2020. 'Open Science Promotes Diverse, Just, and Sustainable Research and Educational Outcomes'. Psychology Learning and Teaching-Plat 19(1):5-20. doi: $10.1177 / 1475725719869164$.

Gregory, Kathleen. 2020. 'Data Discovery and Reuse Practices in Research'.

Gregory, Kathleen, Helena Cousijn, Paul Groth, Andrea Scharnhorst, and Sally Wyatt. 2020. 'Understanding Data Search as a Socio-Technical Practice'. Journal of Information Science 46(4):459-75. doi: 10.1177/0165551519837182.

Gregory, Kathleen, Paul Groth, Helena Cousijn, Andrea Scharnhorst, and Sally Wyatt. 2019. 'Searching Data: A Review of Observational Data Retrieval Practices in Selected Disciplines'. Journal of the Association for Information Science and Technology 70(5):419-32. doi: 10.1002/asi.24165.

Gregory, Kathleen, Paul Groth, Andrea Scharnhorst, and Sally Wyatt. 2020. 'Lost or Found? Discovering Data Needed for Research'. Harvard Data Science Review 2(2). doi: 10.1162/99608f92.e38165eb.

Griesemer, James. 2020. 'A Data Journey Through Dataset-Centric Population Genomics'. Pp. 145-67 in Data Journeys in the Sciences, edited by S. Leonelli and N. Tempini. Cham: Springer International Publishing.

Haak, Wouter, Alberto Zigoni, Helen Kardinaal-de Mooij, and Elena Zudilova-Seinstra. 2020. 'Why Is Getting Credit for Your Data so Hard?' edited by D. Ivanović. ITM Web of Conferences 33:01003. doi: 10.1051/itmconf/20203301003.

Hackett, Edward J., John N. Parker, Niki Vermeulen, and Bart Penders. 2017. 'The Social and Epistemic Organization of Scientific Work'. Pp. 733-64 in The Handbook of Science and Technology Studies. Fourth Edition, edited by U. Felt, R. Fouché, C. A. Miller, and L. Smith-Doerr. Cambridge, MA, London: The MIT Press.

Haeussler, Carolin. 2011. 'Information-Sharing in Academia and the Industry: A Comparative Study'. Research Policy 40(1):105-22. doi: 10.1016/j.respol.2010.08.007. 
Halfmann, Gregor. 2020. 'Material Origins of a Data Journey in Ocean Science: How Sampling and Scaffolding Shape Data Practices'. Pp. 27-44 in Data Journeys in the Sciences, edited by S. Leonelli and N. Tempini. Cham: Springer International Publishing.

Hanson, Brooks, Andrew Sugden, and Bruce Alberts. 2011. 'Making Data Maximally Available'. Science 331(6018):649. doi: 10.1126/science.1203354.

Harding, Sandra. 2002. 'Must the Advance of Science Advance Global Inequality?' International Studies Review 4(2):87-105. doi: 10.1111/1521-9488.00256.

Hey, Tony. 2012. 'The Fourth Paradigm - Data-Intensive Scientific Discovery'. Pp. 1-1 in EScience and Information Management, Communications in Computer and Information Science, edited by S. Kurbanoğlu, U. Al, P. L. Erdoğan, Y. Tonta, and N. Uçak. Berlin, Heidelberg: Springer.

Higman, Rosie, and Stephen Pinfield. 2015. 'Research Data Management and Openness'. Program: Electronic Library and Information Systems 49(4):364-81. doi: 10.1108/PROG-01-2015-0005.

Hillyer, Rebecca, Alejandro Posada, Denisse Albornoz, Leslie Chan, and Angela Okune. 2017. 'Framing a Situated and Inclusive Open Science: Emerging Lessons from the Open and Collaborative Science in Development Network'. in Expanding Perspectives on Open Science: Communities, Cultures and Diversity in Concepts and Practices.

Hine, Christine. 2016. 'Databases as Scientific Instruments and Their Role in the Ordering of Scientific Work': Social Studies of Science 36(2):269-98. doi: 10.1177/0306312706054047.

Hoeppe, Götz. 2020. 'Sharing Data, Repairing Practices: On the Reflexivity of Astronomical Data Journeys'. Pp. 171-90 in Data Journeys in the Sciences, edited by S. Leonelli and N. Tempini. Cham: Springer International Publishing.

Hsu, Leslie, Raleigh L. Martin, Brandon McElroy, and Kimberly Litwin-Miller. 2015. 'Data Management, Sharing, and Reuse in Experimental Geomorphology: Challenges, Strategies, and Scientific Opportunities'. Geomorphology 244:180-89. doi: 10.1016/j.geomorph.2015.03.039.

Hunt, Shanda L., and Caitlin J. Bakker. 2018. 'A Qualitative Analysis of the Information Science Needs of Public Health Researchers in an Academic Setting'. Journal of the Medical Library Association 106(2):184-97. doi: 10.5195/jmla.2018.316.

Ibanez, L., R. Avila, and S. Aylward. 2006. 'Open Source and Open Science: How It Is Changing the Medical Imaging Community'. Pp. 690-93 in 3rd IEEE International Symposium on Biomedical Imaging: Nano to Macro, 2006.

Johnson, Jeffrey Alan. 2014. 'From Open Data to Information Justice'. Ethics and Information Technology 16(4):263-74. doi: 10.1007/s10676-014-9351-8.

Johnson, Jeffrey Alan. 2018. 'Open Data, Big Data, and Just Data'. Public Administration and Information Technology 33:23-49. doi: 10.1007/978-3-319-70894-2_2.

Joo, Soohyung, Sujin Kim, and Youngseek Kim. 2017. 'An Exploratory Study of Health Scientists' Data Reuse Behaviors: Examining Attitudinal, Social, and Resource Factors'. Aslib Journal of Information Management 69(4):389-407. doi: 10.1108/AJIM12-2016-0201. 
Joshi, Margi, and Sharon S. Krag. 2010. 'Issues in Data Management'. Science and Engineering Ethics 16(4):743-48. doi: 10.1007/s11948-010-9223-5.

Karaca, Koray. 2020. 'What Data Get to Travel in High Energy Physics? The Construction of Data at the Large Hadron Collider'. Pp. 45-58 in Data Journeys in the Sciences, edited by S. Leonelli and N. Tempini. Cham: Springer International Publishing.

Kim, Youngseek, and Jeffrey M. Stanton. 2016. 'Institutional and Individual Factors Affecting Scientists' Data-Sharing Behaviors: A Multilevel Analysis'. Journal of the Association for Information Science and Technology 67(4):776-99. doi: 10.1002/asi.23424.

Kim, Youngseek, and Ping Zhang. 2015. 'Understanding Data Sharing Behaviors of STEM Researchers: The Roles of Attitudes, Norms, and Data Repositories'. Library \& Information Science Research 37(3):189-200. doi: 10.1016/j.lisr.2015.04.006.

Kitchin, Rob. 2013. 'Four Critiques of Open Data Initiatives'. Impact of Social Sciences. Retrieved 7 May 2020 (https://blogs.Ise.ac.uk/impactofsocialsciences/2013/11/27/fourcritiques-of-open-data-initiatives/).

Kitchin, Rob. 2014. 'Big Data, New Epistemologies and Paradigm Shifts'. Big Data \& Society 1(1):2053951714528481. doi: 10.1177/2053951714528481.

Knorr Cetina, Karin. 1981. The Manufacture of Knowledge. An Essay on the Constructivist and Contextual Nature of Science. Oxford: Pergamon Press.

Knorr Cetina, Karin. 1991. 'Epistemic Cultures: Forms of Reason in Science'. History of Political Economy 23(1):105-22.

Knorr Cetina, Karin. 1995. 'Laboratory Studies. The Cultural Approach to the Study of Science'. Pp. 140-66 in Handbook of Science and Technology Studies. Thousand Oaks, London, New Delhi: SAGE Publications.

Knorr Cetina, Karin. 1999. Epistemic Cultures. How the Sciences Make Knowledge. 1st ed. Cambridge, Massachusetts: Harvard University Press.

Knorr Cetina, Karin. 2007. 'Culture in Global Knowledge Societies: Knowledge Cultures and Epistemic Cultures'. Interdisciplinary Science Reviews 32(4):361-75. doi: 10.1179/030801807X163571.

Koopman, Margaret M., and Karin de Jager. 2016. 'Archiving South African Digital Research Data: How Ready Are We?' South African Journal of Science 112(7/8):7. doi: 10.17159/sajs.2016/20150316.

Kuhn, Thomas S. 2012. The Structure of Scientific Revolutions. Fourth edition. Chicago, London: The University of Chicago Press.

Kurata, Keiko, Mamiko Matsubayashi, and Shinji Mine. 2017. 'Identifying the Complex Position of Research Data and Data Sharing Among Researchers in Natural Science'. Sage Open 7(3):2158244017717301. doi: 10.1177/2158244017717301.

Lamont, Michele. 2009. How Professors Think. Inside the Curious World of Academic Judgement. Cambridge, MA; London: Havard University Press.

Latour, Bruno. 1994. Science in Action. How to Follow Scientists and Engineers through Society. 6. Aufl. Cambridge, Mass.: Harvard University Press. 
Latour, Bruno, and Steve Woolgar. 1986. Laboratory Life. The Construction of Scientific Facts. Princeton, NJ: Princeton University Press.

Leonelli, Sabina. 2016. Data-Centric Biology. A Philosophical Study. Chicago, London: The University of Chicago Press.

Leonelli, Sabina. 2017. "Global Data Quality Assessment and the Situated Nature of "Best" Research Practices in Biology'. Data Science Journal 16(0):32. doi: 10.5334/dsj-2017032.

Leonelli, Sabina. 2018. 'Re-Thinking Reproducibility as a Criterion for Research Quality'. Pp. 129-46 in Including a Symposium on Mary Morgan: Curiosity, Imagination, and Surprise. Vol. 36B, Research in the History of Economic Thought and Methodology. Emerald Publishing Limited.

Leonelli, Sabina. 2020. 'Learning from Data Journeys'. Pp. 1-24 in Data Journeys in the Sciences, edited by S. Leonelli and N. Tempini. Cham: Springer International Publishing.

Leonelli, Sabina, Nicholas Smirnoff, Jonathan Moore, Charis Cook, and Ruth Bastow. 2013. 'Making Open Data Work for Plant Scientists'. Journal of Experimental Botany 64(14):4109-17. doi: 10.1093/jxb/ert273.

Leonelli, Sabina, Daniel Spichtinger, and Barbara Prainsack. 2015. 'Sticks and Carrots: Encouraging Open Science at Its Source'. Geo 2(1):12-16. doi: 10.1002/geo2.2.

Levin, Nadine, and Sabina Leonelli. 2017. "How Does One "Open" Science? Questions of Value in Biological Research'. Science, Technology, \& Human Values 42(2):280-305. doi: $10.1177 / 0162243916672071$.

Lilja, Erika. 2020. 'Threat of Policy Alienation: Exploring the Implementation of Open Science Policy in Research Practice'. Science and Public Policy (scaa044). doi: 10.1093/scipol/scaa044.

Littler, Katherine, Wee-Ming Boon, Gail Carson, and Evelyn Depoortere. 2017. 'Progress in Promoting Data Sharing in Public Health Emergencies'. Bulletin of the World Health Organization 95(4):243-243A. doi: 10.2471/BLT.17.192096.

Liu, Xia, and Ning Ding. 2016. 'Research Data Management in Universities of Central China'. The Electronic Library. doi: 10.1108/EL-04-2015-0063.

Lynch, Michael. 1985. Art and Artifact in Laboratory Practice. A Study of Shop Work and Shop Talk in a Research Laboratory. London, New York: Routledge and Kegan Paul.

Maasen, Sabine, and Oliver Lieven. 2006. 'Transdisciplinarity: A New Mode of Governing Science?' Science and Public Policy 33:399-410.

Maisuria, Alpesh, and Mike Cole. 2017. 'The Neoliberalization of Higher Education in England: An Alternative Is Possible'. Policy Futures in Education 15(5):602-19. doi: 10.1177/1478210317719792.

Mallasvik, Mari Louise, and Jorge Tiago Martins. 2021. 'Research Data Sharing Behaviour of Engineering Researchers in Norway and the UK: Uncovering the Double Face of Janus'. Journal of Documentation 77(2):576-93. doi: 10.1108/JD-08-2020-0135. 
Mancilla, Heather Andrews, Marta Teperek, Jasper van Dijck, Kees den Heijer, Robbert Eggermont, Esther Plomp, Yasemin Turkyilmaz-van der Velden, and Shalini Kurapati. 2019. 'On a Quest for Cultural Change - Surveying Research Data Management Practices at Delft University of Technology'. Liber Quarterly: The Journal of European Research Libraries 29(1):1-27. doi: 10.18352/lq.10287.

Manzi, M., D. Ojeda, and R. Hawkins. 2019. “"Enough Wandering Around!”: Life Trajectories, Mobility, and Place Making in Neoliberal Academia'. Professional Geographer 71(2):355-63. doi: 10.1080/00330124.2018.1531036.

Mauthner, Natasha Susan, and Odette Parry. 2013. 'Open Access Digital Data Sharing: Principles, Policies and Practices'. Social Epistemology 27(1):47-67. doi: 10.1080/02691728.2012.760663.

Mayer, Katja, Katharina Rieck, Stefan Reichmann, Patrick Danowski, Anton Graschopf, Thomas König, Peter Kraker, Patrick Lehner, Falk Reckling, Tony Ross-Hellauer, Daniel Spichtinger, Michalis Tzatzanis, and Stefanie Schürz. 2020. 'Empfehlungen Für Eine Nationale Open Science Strategie in Österreich / Recommendations for a National Open Science Strategy in Austria'. doi: 10.5281/zenodo.4109242.

Mayernik, Matthew S. 2016. 'Research Data and Metadata Curation as Institutional Issues'. Journal of the Association for Information Science and Technology 67(4):973-93. doi: https://doi.org/10.1002/asi.23425.

Mayernik, Matthew S. 2019. 'Metadata Accounts: Achieving Data and Evidence in Scientific Research'. Social Studies of Science 49(5):732-57. doi: 10.1177/0306312719863494.

Mayernik, Matthew S., Archer L. Batcheller, and Christine L. Borgman. 2011. 'How Institutional Factors Influence the Creation of Scientific Metadata'. Pp. 417-25 in Proceedings of the 2011 iConference, iConference '11. New York, NY, USA: Association for Computing Machinery.

McKiernan, Erin C., Philip E. Bourne, C. Titus Brown, and Stuart Buck. 2016. 'How Open Science Helps Researchers Succeed'. ELife 5. doi: 10.7554/eLife.16800.

Merton, Robert K. 1968. 'The Matthew Effect in Science: The Reward and Communication Systems of Science Are Considered'. Science 159(3810):56-63. doi: 10.1126/science.159.3810.56.

Merton, Robert K. 1973. 'The Normative Structure of Science'. Pp. 267-80 in The Sociology of Science. Chicago: The University of Chicago Press.

Merton, Robert K. 1988. 'The Matthew Effect in Science, II. Cumulative Advantage and the Symbolism of Intellectual Property'. Isis 79:606-23.

Mirowski, Philip. 2018. 'The Future(s) of Open Science'. Social Studies of Science 48(2):171203. doi: https://doi.org/10.1177/0306312718772086.

Mirowski, Philip, and Esther-Mirjam Sent. 2002. Science Bought and Sold: Essays in the Economics of Science. University of Chicago Press.

Morley, Louise, Nafsika Alexiadou, Stela Garaz, José González-Monteagudo, and Marius Taba. 2018. 'Internationalisation and Migrant Academics: The Hidden Narratives of Mobility'. Higher Education 76(3):537-54. doi: 10.1007/s10734-017-0224-z. 
Mosconi, Gaia, Qinyu Li, Dave Randall, Helena Karasti, Peter Tolmie, Jana Barutzky, Matthias Korn, and Volkmar Pipek. 2019. 'Three Gaps in Opening Science'. Computer Supported Cooperative Work (CSCW) 28(3):749-89. doi: 10.1007/s10606-019-09354$\mathrm{Z}$.

Münch, Richard. 2014. Academic Capitalism. London, New York: Routledge.

Murillo, Angela P. 2016. 'How Do Scientists Determine Data Reusability? A Quasi-Experiment Think-Aloud Study: How Do Scientists Determine Data Reusability? A QuasiExperiment Think-Aloud Study'. Proceedings of the Association for Information Science and Technology 53(1):1-6. doi: 10.1002/pra2.2016.14505301110.

Murillo, Angela P. 2019. 'Data Matters: How Earth and Environmental Scientists Determine Data Relevance and Reusability'. Collection and Curation ahead-of-print(ahead-ofprint). doi: 10.1108/CC-11-2018-0023.

Myneni, Sahiti, Vimla L. Patel, G. Steven Bova, Jian Wang, Christopher F. Ackerman, Cynthia A. Berlinicke, Steve H. Chen, Mikael Lindvall, and Donald J. Zack. 2016. 'Resolving Complex Research Data Management Issues in Biomedical Laboratories: Qualitative Study of an Industry-Academia Collaboration'. Computer Methods and Programs in Biomedicine 126:160-70. doi: 10.1016/j.cmpb.2015.11.001.

Nielsen, Michael. 2013. Reinventing Discovery: The New Era of Networked Science. Princeton: Princeton University Press.

Nosek, B. A., G. Alter, G. C. Banks, D. Borsboom, S. D. Bowman, S. J. Breckler, S. Buck, C. D. Chambers, G. Chin, G. Christensen, M. Contestabile, A. Dafoe, E. Eich, J. Freese, R. Glennerster, D. Goroff, D. P. Green, B. Hesse, M. Humphreys, J. Ishiyama, D. Karlan, A. Kraut, A. Lupia, P. Mabry, T. Madon, N. Malhotra, E. Mayo-Wilson, M. McNutt, E. Miguel, E. Levy Paluck, U. Simonsohn, C. Soderberg, B. A. Spellman, J. Turitto, G. VandenBos, S. Vazire, E. J. Wagenmakers, R. Wilson, and T. Yarkoni. 2015. 'Promoting an Open Research Culture'. Science 348(6242):1422-25. doi: 10.1126/science.aab2374.

Olesk, Arko, Esta Kaal, and kristel Toom. 2019. 'The Possibilities of Open Science for Knowledge Transfer in the Science-Policy Interface'. Journal of Science Communication 18(3). doi: 10.22323/2.18030203.

Olmos-Peñuela, Julia, Paul Benneworth, and Elena Castro-Martínez. 2015. 'What Stimulates Researchers to Make Their Research Usable? Towards an "Openness" Approach'. Minerva 53(4):381-410. doi: 10.1007/s11024-015-9283-4.

Park, Min Sook, and Hyoungjoo Park. 2019. 'An Examination of Metadata Practices for Research Data Reuse: Characteristics and Predictive Probability of Metadata Elements'. Malaysian Journal of Library \& Information Science 24(3):61-75. doi: $10.22452 / \mathrm{mjlis} . v o l 24$ no3.4.

Pasquetto, Irene V., Bernadette M. Randles, and Christine L. Borgman. 2017. 'On the Reuse of Scientific Data'. Data Science Journal 16:8. doi: 10.5334/dsj-2017-008.

Perrier, Laure, Erik Blondal, A. Patricia Ayala, and Dylanne Dearborn. 2017. 'Research Data Management in Academic Institutions: A Scoping Review'. PloS One 12(5):e0178261.

Pisani, Elizabeth, James Whitworth, Basia Zaba, and Carla Abou-Zahr. 2010. 'Time for Fair Trade in Research Data'. Lancet (London, England) 375(9716):703-5. doi: 10.1016/S0140-6736(09)61486-0. 
Piwowar, Heather A. 2011. 'Who Shares? Who Doesn't? Factors Associated with Openly Archiving Raw Research Data'. PLOS ONE 6(7):e18657. doi: 10.1371/journal.pone.0018657.

Piwowar, Heather A., and Wendy W. Chapman. 2010. 'Public Sharing of Research Datasets: A Pilot Study of Associations'. Journal of Informetrics 4(2):148-56. doi: 10.1016/j.joi.2009.11.010.

Piwowar, Heather A., Roger S. Day, and Douglas B. Fridsma. 2007. 'Sharing Detailed Research Data Is Associated with Increased Citation Rate'. PLOS ONE 2(3):e308. doi: 10.1371/journal.pone.0000308.

Piwowar, Heather A., and Todd J. Vision. 2013a. 'Data Reuse and the Open Data Citation Advantage'. PeerJ 1:e175. doi: 10.7717/peerj.175.

Piwowar, Heather A., and Todd J. Vision. 2013b. 'Data Reuse and the Open Data Citation Advantage'. PeerJ 1:e175. doi: 10.7717/peerj.175.

Pöschl, Ulrich. 2012. 'Multi-Stage Open Peer Review: Scientific Evaluation Integrating the Strengths of Traditional Peer Review with the Virtues of Transparency and SelfRegulation'. Frontiers in Computational Neuroscience 6. doi: 10.3389/fncom.2012.00033.

Poth, Cheryl N. 2019. 'Rigorous and Ethical Qualitative Data Reuse: Potential Perils and Promising Practices'. International Journal of Qualitative Methods 18:UNSP 1609406919868870. doi: 10.1177/1609406919868870.

Ram, Karthik. 2013. 'Git Can Facilitate Greater Reproducibility and Increased Transparency in Science'. Source Code for Biology and Medicine 8(1):7. doi: 10.1186/1751-0473-8-7.

Rappert, Brian, and Louise Bezuidenhout. 2016. 'Data Sharing in Low-Resourced Research Environments'. Prometheus 34(3-4):207-24. doi: 10.1080/08109028.2017.1325142.

Reichmann, Stefan, Thomas Klebel, Ilire Hasani-Mavriqi, and Tony Ross-Hellauer. 2021. 'Between Administration and Research: Understanding Data Management Practices in an Institutional Context'. Journal of the Association for Information Science and Technology 11(72):1415-31. doi: https://doi.org/10.1002/asi.24492.

Richardson, Julie, and Diane Hoffman-Kim. 2010. 'The Importance of Defining "Data" in Data Management Policies'. Science and Engineering Ethics 16(4):749-51. doi: 10.1007/s11948-010-9231-5.

Richterich, Annika. 2018. The Big Data Agenda: Data Ethics and Critical Data Studies. University of Westminster Press.

Ross-Hellauer, Tony. 2017. 'What Is Open Peer Review? A Systematic Review'. F1000Research 6:588. doi: 10.12688/f1000research.11369.2.

Ross-Hellauer, Tony, Arvid Deppe, and Birgit Schmidt. 2017. 'Survey on Open Peer Review: Attitudes and Experience amongst Editors, Authors and Reviewers' edited by J. M. Wicherts. PLOS ONE 12(12):e0189311. doi: 10.1371/journal.pone.0189311.

Ross-Hellauer, Tony, Stefan Reichmann, Nicki Lisa Cole, Angela Fessl, Thomas Klebel, and Nancy Pontika. 2022. 'Dynamics of Cumulative Advantage and Threats to Equity in Open Science: A Scoping Review'. Royal Society Open Science 9(1):211032. doi: $10.1098 /$ rsos. 211032 . 
Roth, Wolff-Michael, and G. Michael Bowen. 1999. 'Digitizing Lizards: The Topology of 'Vision' in Ecological Fieldwork'. Social Studies of Science 29(5):719-64. doi: 10.1177/030631299029005003.

Sautier, Marie. 2021. 'Move or Perish? Sticky Mobilities in the Swiss Academic Context'. Higher Education 82(4):799-822. doi: 10.1007/s10734-021-00722-7.

Schaeffer, Veronique. 2019. 'The Use of Material Transfer Agreements in Academia: A Threat to Open Science or a Cooperation Tool?' Research Policy 48(9):UNSP 103824. doi: 10.1016/j.respol.2019.103824.

Schöpfel, Joachim, Coline Ferrant, Francis André, and Renaud Fabre. 2018. 'Research Data Management in the French National Research Center (CNRS)'. Data Technologies and Applications. doi: 10.1108/DTA-01-2017-0005.

Shanahan, Daniel R., and Bjorn R. Olsen. 2014. 'Opening Peer-Review: The Democracy of Science'. Journal of Negative Results in BioMedicine 13(1):2. doi: 10.1186/1477-575113-2.

Slaughter, Sheila, and Gary Rhoades. 2000. 'The Neo-Liberal University'. New Labor Forum (6):73-79.

Slaughter, Sheila, and Gary Rhoades. 2010. Academic Capitalism and the New Economy. Markets, State, and Higher Education. Baltimore, MD: The Johns Hopkins University Press.

Stichweh, Rudolf. 1994. Wissenschaft, Universität, Professionen: soziologische Analysen. 1. Aufl. Frankfurt am Main: Suhrkamp.

Suber, Peter. 2012. Open Access. MIT Press. Cambridge, MA.

Tennant, Jonathan, Damien Jacques, and Lauren Collister. 2016. 'The Academic, Economic and Societal Impacts of Open Access: An Evidence-Based Review' edited by L. Collister. F1000Research 5. doi: 10.12688/f1000research.8460.1.

Tennant, Jonathan P., and Tony Ross-Hellauer. 2020. 'The Limitations to Our Understanding of Peer Review'. Research Integrity and Peer Review 5(1):6. doi: 10.1186/s41073-02000092-1.

Tenopir, Carol, Suzie Allard, Kimberly Douglass, Arsev Umur Aydinoglu, Lei Wu, Eleanor Read, Maribeth Manoff, and Mike Frame. 2011. 'Data Sharing by Scientists: Practices and Perceptions'. PLOS ONE 6(6):e21101. doi: 10.1371/journal.pone.0021101.

Tenopir, Carol, Natalie M. Rice, Suzie Allard, Lynn Baird, Josh Borycz, Lisa Christian, Bruce Grant, Robert Olendorf, and Robert J. Sandusky. 2020. 'Data Sharing, Management, Use, and Reuse: Practices and Perceptions of Scientists Worldwide'. PLOS ONE 15(3):e0229003. doi: 10.1371/journal.pone.0229003.

Thanos, Costantino. 2017. 'Research Data Reusability: Conceptual Foundations, Barriers and Enabling Technologies'. Publications 5(1):2. doi: 10.3390/publications5010002.

Thylstrup, Nanna Bonde. 2019. 'Data out of Place: Toxic Traces and the Politics of Recycling'. Big Data \& Society 6(2):2053951719875479. doi: 10.1177/2053951719875479. 
Tkacz, Nathaniel, Mário Henrique da Mata Martins, João Porto de Albuquerque, Flávio Horita, and Giovanni Dolif Neto. 2021. 'Data Diaries: A Situated Approach to the Study of Data'. Big Data \& Society 8(1):2053951721996036. doi: 10.1177/2053951721996036.

Toelch, Ulf, and Dirk Ostwald. 2018. 'Digital Open Science-Teaching Digital Tools for Reproducible and Transparent Research'. PLOS Biology 16(7):e2006022. doi: 10.1371/journal.pbio.2006022.

Tyfield, David. 2013. 'Transition to Science 2.0: "Remoralizing" the Economy of Science'. Spontaneous Generations: A Journal for the History and Philosophy of Science 7(1):29-48. doi: 10.4245/sponge.v7i1.19664.

Vale, Ronald D., and Anthony A. Hyman. 2016. 'Priority of Discovery in the Life Sciences'. ELife 5:e1693. doi: 10.7554/eLife.16931.

Vicente-Saez, Ruben, and Clara Martinez-Fuentes. 2018. 'Open Science Now: A Systematic Literature Review for an Integrated Definition'. Journal of Business Research 88:42836. doi: 10.1016/j.jbusres.2017.12.043.

Vogeli, Christine, Recai Yucel, Eran Bendavid, Lisa M. Jones, Melissa S. Anderson, Karen Seashore Louis, and Eric G. Campbell. 2006. 'Data Withholding and the next Generation of Scientists: Results of a National Survey'. Academic Medicine: Journal of the Association of American Medical Colleges 81(2):128-36.

Wade, Ted D. 2014. 'Refining Gold from Existing Data'. Current Opinion in Allergy and Clinical Immunology 14(3):181-85. doi: 10.1097/ACI.0000000000000051.

Wallis, Jillian C., and Christine L. Borgman. 2011. 'Who Is Responsible for Data? An Exploratory Study of Data Authorship, Ownership, and Responsibility'. Proceedings of the American Society for Information Science and Technology 48(1):1-10. doi: 10.1002/meet.2011.14504801188.

Wallis, Jillian C., Matthew S. Mayernik, Christine L. Borgman, and Alberto Pepe. 2010. 'Digital Libraries for Scientific Data Discovery and Reuse: From Vision to Practical Reality'. P. 333 in Proceedings of the 10th annual joint conference on Digital libraries - JCDL '10. Gold Coast, Queensland, Australia: ACM Press.

Wallis, Jillian C., Elizabeth Rolando, and Christine L. Borgman. 2013. 'If We Share Data, Will Anyone Use Them? Data Sharing and Reuse in the Long Tail of Science and Technology'. PLOS ONE 8(7):e67332. doi: 10.1371/journal.pone.0067332.

Wan, Xiang, and Paul Pavlidis. 2007. 'Sharing and Reusing Gene Expression Profiling Data in Neuroscience'. Neuroinformatics 5(3):161-75. doi: 10.1007/s12021-007-0012-5.

White, Ethan, Elita Baldridge, Zachary Brym, Kenneth Locey, Daniel McGlinn, and Sarah Supp. 2013. 'Nine Simple Ways to Make It Easier to (Re)Use Your Data'. Ideas in Ecology and Evolution 6(2). doi: 10.4033/iee.2013.6b.6.f.

Whitlock, Michael C., Mark A. McPeek, Mark D. Rausher, Loren Rieseberg, and Allen J. Moore. 2010. 'Data Archiving.' The American Naturalist 175(2):145-46. doi: 10.1086/650340.

Whyte, Angus, and Jonathan Tedds. 2011. 'Making the Case for Research Data Management'.

Wilkinson, Mark D., Michel Dumontier, IJsbrand Jan Aalbersberg, Gabrielle Appleton, Myles Axton, Arie Baak, Niklas Blomberg, Jan-Willem Boiten, Luiz Bonino da Silva Santos, Philip E. Bourne, Jildau Bouwman, Anthony J. Brookes, Tim Clark, Mercè Crosas, 
Ingrid Dillo, Olivier Dumon, Scott Edmunds, Chris T. Evelo, Richard Finkers, Alejandra Gonzalez-Beltran, Alasdair J. G. Gray, Paul Groth, Carole Goble, Jeffrey S. Grethe, Jaap Heringa, Peter A. C. 't Hoen, Rob Hooft, Tobias Kuhn, Ruben Kok, Joost Kok, Scott J. Lusher, Maryann E. Martone, Albert Mons, Abel L. Packer, Bengt Persson, Philippe Rocca-Serra, Marco Roos, Rene van Schaik, Susanna-Assunta Sansone, Erik Schultes, Thierry Sengstag, Ted Slater, George Strawn, Morris A. Swertz, Mark Thompson, Johan van der Lei, Erik van Mulligen, Jan Velterop, Andra Waagmeester, Peter Wittenburg, Katherine Wolstencroft, Jun Zhao, and Barend Mons. 2016. 'The FAIR Guiding Principles for Scientific Data Management and Stewardship'. Scientific Data 3:160018.

Wilkinson, Mark D., Susanna-Assunta Sansone, Erik Schultes, Peter Doorn, Luiz Olavo Bonino da Silva Santos, and Michel Dumontier. 2018. 'A Design Framework and Exemplar Metrics for FAIRness'. Scientific Data 5:180118. doi: 10.1038/sdata.2018.118.

Wu, Shuheng, and Adam Worrall. 2019. 'Supporting Successful Data Sharing Practices in Earthquake Engineering'. Library Hi Tech 37(4):764-80. doi: 10.1108/LHT-03-20190058.

Yoon, Ayoung. 2017. 'Data Reusers' Trust Development'. Journal of the Association for Information Science and Technology 68(4):946-56. doi: 10.1002/asi.23730.

Yoon, Ayoung, Wei Jeng, Renata Curty, and Angela Murillo. 2017. 'In between Data Sharing and Reuse: Shareability, Availability and Reusability in Diverse Contexts'. Proceedings of the Association for Information Science and Technology 54(1):606-9. doi: 10.1002/pra2.2017.14505401085.

Yoon, Ayoung, and Youngseek Kim. 2017. 'Social Scientists' Data Reuse Behaviors: Exploring the Roles of Attitudinal Beliefs, Attitudes, Norms, and Data Repositories'. Library \& Information Science Research 39(3):224-33. doi: 10.1016/j.lisr.2017.07.008.

Yoon, Ayoung, and Youngseek Kim. 2020. 'The Role of Data-Reuse Experience in Biological Scientists' Data Sharing: An Empirical Analysis'. Electronic Library 38(1):186-208. doi: 10.1108/EL-06-2019-0146.

Zimmerman, Ann S. 2008. 'New Knowledge from Old Data: The Role of Standards in the Sharing and Reuse of Ecological Data'. Science, Technology, \& Human Values 33(5):631-52. doi: 10.1177/0162243907306704.

Zuboff, Shoshana. 2019. The Age of Surveillance Capitalism: The Fight for a Human Future at the New Frontier of Power. Paperback edition. London: Profile Books.

Zuckerman, Harriet. 1988. 'The Sociology of Science'. Pp. 511-74 in Handbook of sociology, edited by N. J. Smelser. Thousand Oaks, CA, US: Sage Publications, Inc. 